\title{
Spectral correlations and antimicrobial activities of some 1-pyrenyl chalcones
}

\author{
R. Arulkumaran ${ }^{1}$, S. Vijayakumar ${ }^{1}$, R. Sundararajan ${ }^{1}$, S. P. Sakthinathan ${ }^{1}$, \\ D. Kamalakkannan ${ }^{1}$, R. Suresh ${ }^{1}$, K. Ranganathan ${ }^{1}$, P. R. Rajakumar ${ }^{1}$, \\ G. Vanangamudi ${ }^{1}$, G. Thirunarayanan ${ }^{2, *}$ \\ ${ }^{1}$ PG \& Research Department of Chemistry, Government Arts College, C-Mutlur, \\ Chidambaram - 608102, India. \\ ${ }^{2}$ Department of Chemistry, Annamalai University, Annamalainagar - 608002, India. \\ *E-mail address: drgtnarayanan@gmail.com
}

\begin{abstract}
A series of 1-pyrenyl chalcones have been synthesized by Crossed-Aldol condensation of 1acetylpyrene and substituted benzaldehydes. The purities of these chalcones have been checked by their physical constants, UV, IR, NMR and MASS spectral data. The spectral data of these chalcones have been correlated with Hammett sigma constants, $\mathrm{F}$ and $\mathrm{R}$ parameters using single and multi-linear regression analysis. From the results of statistical analysis, the effect of substituents on the spectral group frequencies have been discussed. The anti-microbial activities of these chalcones have been evaluated using Bauer-Kirby method.
\end{abstract}

Keywords: Substituted styryl 1-pyrenyl ketones; UV spectra; IR spectra; NMR spectra; Substituent effects; Antimicrobial activities

\section{INTRODUCTION}

The $\sigma, \beta$ unsaturated ketones are called as enones. They possess carbonyl moiety and it was bonded with aryl or alkyl groups in both sides. They are very important molecules for synthesising heterocycles and possess multipronged activities [1]. Various solvent assisted and green synthetic methodologies have been reported for the synthesis of aryl enones. The Crossed-Aldol [2], Claisen Schmidt [3], Knovenagal [4] and Wittig [5] reactions are important for the synthesis of enones. Many catalyst were used for proceedings the above said reactions namely, EtOH-NaOH [6], $\mathrm{MeOH}-\mathrm{KOH}$ [7], EtOH-KOH [8], $\mathrm{MgCl}_{2}$ [5], silica-sulphuric acid [9], anhydrous zinc chloride [10], clay [11], Hydrotalcite [12], ground chemistry catalysts-grinding the reactants with sodium hydroxide [13], aqueous alkali in lower temperature [14], solid sulphonic acid from bamboo [15], barium hydroxide [16] anhydrous sodium bicarbonate [17], microwave assisted synthesis [18], flyash: water [19], fly-ash: $\mathrm{H}_{2} \mathrm{SO}_{4}$ [20], fly-ash: PTS [21], $\mathrm{NaOH}-\mathrm{CTABr}$ [22], $\mathrm{SiO}_{2}-\mathrm{H}_{3} \mathrm{PO}_{4}$ [23], $\mathrm{SOCl}_{2}$ [24] and sulfated titania [25]. The spectral data of these E- chalcones were useful for prediction of ground state equilibration like s-cis and s-trans conformers. Recently, Janaki et al., [23] have elaborately studied the effects of substituent on the ultraviolet absorption $(\lambda$ max, $\mathrm{nm})$, infrared group frequencies $\left(\nu, \mathrm{cm}^{-1}\right)$ and NMR chemical shifts $(\delta, \mathrm{ppm})$ of $\sigma, \beta$-proton and carbons of 4-ethoxyphenyl chalcones and benzimidazole chalcones. In their studies, they observed satisfactory and good correlations for each spectral data with Hammett substituent constants, F and $\mathrm{R}$ parameters. Chalcones possess various multipronged activities such as antimicrobial [26], antidepressants [27], antiplosmodial [28], anti-aids [29] and insect antifeedant activities [13,22]. In the present investigation, the authors have synthesized a series of some substituted styryl 1-pyrenyl 
ketones, studied the spectral correlations and to evaluate the antimicrobial activities using BauerKirby [30] method.

\section{EXPERIMENTAL}

\section{1. General}

All chemicals used were purchased from Sigma-Aldrich and E-Merck chemical companies. Melting points of all chalcones were determined in open glass capillaries on Mettler FP51 melting point apparatus and are uncorrected. The ELICO BL $222 \quad$ ultraviolet spectrophotometer was utilized for recording the absorption maxima $(\lambda \max , \mathrm{nm})$, of all chalcones in spectral grade methanol. Infrared spectra (KBr, 4000-400 $\mathrm{cm}^{-1}$ ) were recorded AVATAR-300 Fourier transform spectrophotometer. Bruker AV400 NMR spectrometer was used for recording NMR spectra operating at $400 \mathrm{MHz}$ for ${ }^{1} \mathrm{H}$ spectra and $125.46 \mathrm{MHz}$ for ${ }^{13} \mathrm{C}$ spectra in $\mathrm{CDCl}_{3}$ solvent using TMS as internal standard. Mass spectra of all chalcones were recorded on SHIMADZU spectrometer using chemical ionization technique.

\section{2. Synthesis of Chalcones}

All substituted styryl-1-pyrenyl chalcones were synthesized by procedure published in literature. An equi-molar quantities of 1-acetylpyrene $(2 \mathrm{mmol})$ and substituted benzaldehydes $(2$ $\mathrm{mmol})$, Fly-ash(0.5 g) with $20 \mathrm{~mL}$ of water [19, 31] (Scheme 1) were refluxed for $4 \mathrm{~h}$. and the completion of the reaction was monitored by thin layer chromatography. After completion of the reaction, the solid product was separated by filtration. Further the product was purified by column chromatography using dichloromethane and ethyl acetate mixture as eluent. The physical constants, analytical and mass fragments were presented in Table 1.<smiles>CC(=O)c1cc2cccc3ccc4cccc1c4c32</smiles><smiles>[X]c1ccc(C=O)cc1</smiles>

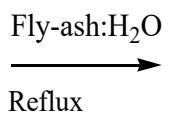<smiles>[X]c1ccc(/C=C/C(=O)c2cc3cccc4ccc5cccc2c5c43)cc1</smiles>

Where $\mathrm{X}=\mathrm{H}, m-\mathrm{Br}, p$ - $\mathrm{Br}, p-\mathrm{Cl}, p-\mathrm{F}, o-\mathrm{CH}_{3}, p-\mathrm{CH}_{3}, m-\mathrm{NO}_{2}, p-\mathrm{NO}_{2}$

Scheme 1. Synthesis of substituted styryl 1-pyrenyl ketones.

Table 1. Physical constants and mass spectral data of substituted styryl 1-pyrenyl ketones.

\begin{tabular}{|c|c|c|c|c|c|}
\hline Entry & $\mathbf{X}$ & $\mathbf{M . ~ F . ~}$ & M. W. & m.p. $\left({ }^{\circ} \mathbf{C}\right)$ & Mass (m/z) \\
\hline 1 & $\mathrm{H}$ & $\mathrm{C}_{25} \mathrm{H}_{16} \mathrm{O}$ & 332 & $122-123(122-123)[31]$ & $332\left[\mathrm{M}^{+}\right]$ \\
\hline 2 & $m-\mathrm{Br}$ & $\mathrm{C}_{25} \mathrm{H}_{15} \mathrm{OBr}$ & 411 & $119-120(119-120)[31]$ & $411\left[\mathrm{M}^{+}\right], 413\left[\mathrm{M}^{2+}\right]$ \\
\hline 3 & $o-\mathrm{Cl}$ & $\mathrm{C}_{25} \mathrm{H}_{15} \mathrm{OCl}$ & 366 & $119-120(119-120)[31]$ & $366\left[\mathrm{M}^{+}\right], 368\left[\mathrm{M}^{2+}\right]$ \\
\hline 4 & $m-\mathrm{Cl}$ & $\mathrm{C}_{25} \mathrm{H}_{15} \mathrm{OCl}$ & 366 & $262-263(262-263)[31]$ & $366\left[\mathrm{M}^{+}\right], 368\left[\mathrm{M}^{2+}\right]$ \\
\hline 5 & $p-\mathrm{Cl}$ & $\mathrm{C}_{25} \mathrm{H}_{15} \mathrm{OCl}$ & 366 & $96-97(96-97)[31]$ & $366\left[\mathrm{M}^{+}\right], 368\left[\mathrm{M}^{2+}\right]$ \\
\hline
\end{tabular}




\begin{tabular}{|c|c|c|c|c|c|}
\hline 6 & $p-\mathrm{F}$ & $\mathrm{C}_{25} \mathrm{H}_{15} \mathrm{OF}$ & 350 & $108-109(108-109)[31]$ & $350\left[\mathrm{M}^{+}\right], 352\left[\mathrm{M}^{2+}\right]$ \\
\hline 7 & $p-\mathrm{CH}_{3}$ & $\mathrm{C}_{26} \mathrm{H}_{18} \mathrm{O}$ & 346 & $219-220(219-220)[31]$ & $346\left[\mathrm{M}^{+}\right]$ \\
\hline 8 & $m-\mathrm{NO}_{2}$ & $\mathrm{C}_{25} \mathrm{H}_{15} \mathrm{NO}_{3}$ & 377 & $128-129(128-129)[31]$ & $377\left[\mathrm{M}^{+}\right]$ \\
\hline 9 & $p-\mathrm{NO}_{2}$ & $\mathrm{C}_{25} \mathrm{H}_{15} \mathrm{NO}_{3}$ & 377 & $136-137(136-137)[31]$ & $377\left[\mathrm{M}^{+}\right]$ \\
\hline
\end{tabular}

\section{RESULTS AND DISCUSSION}

In the present study the authors have studied the effects of substituents on the spectral data such as ultraviolet absorption maxima $(\lambda \max , \mathrm{nm})$, infrared spectral carbonyl and deformation modes $\left(v, \mathrm{~cm}^{-1}\right)$, the chemical shifts $(\delta, \mathrm{ppm})$ of $\alpha, \beta$ protons, and carbonyl carbons of synthesized 1pyrrenyl chalcones with Hammett substituent constants, $\mathrm{F}$ and $\mathrm{R}$ parameters using single and multilinear regression analysis for predicting the reactivity on the group frequencies.

\section{1. UV spectral study}

The measured ultraviolet absorption maxima $(\lambda \max , \mathrm{nm})$ of all 1-pyrenyl chalcones were tabulated in Table 2. These values are correlated [1, 2, 6, 10, 19, 21, 23-25, 28, 32] with Hammett substituent constants, $\mathrm{F}$ and $\mathrm{R}$ parameters using single and multi-linear regression analysis. While seeking Hammett correlation, involving UV absorption maximum values, the form of the Hammett equation employed is as given in equation.

$$
\lambda \max =\rho \sigma+\lambda_{0}
$$

The results of statistical analysis are presented in Table 3. From the Table 3, the correlation of absorption maxima $(\lambda \max , \mathrm{nm})$ with $\mathrm{F}$ parameter is satisfactory. The remaining Hammett substituent constants and $\mathrm{R}$ parameter were failing in correlation. 
Table 2. The ultraviolet absorption maxima $(\lambda \max , \mathrm{nm})$, infrared absorptions $\left(v, \mathrm{~cm}^{-1}\right)$ and NMR chemical shifts $(\delta$, ppm) of substituted styryl 1-pyrenyl ketones.

\begin{tabular}{|c|c|c|c|c|c|c|c|c|c|c|c|c|c|}
\hline 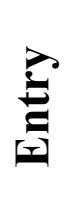 & $x$ & है & 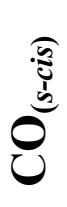 & $\overbrace{0}^{\overparen{E}}$ & $\stackrel{s}{0}$ & $\frac{5}{0}$ & 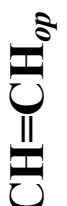 & $\bigcup_{0}^{\overline{8}}$ & 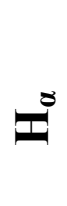 & Iี & $ن^{\circ}$ & $\tilde{u}$ & ن \\
\hline- & $I$ & $\begin{array}{l}8 \\
\stackrel{0}{0} \\
\text { సे }\end{array}$ & 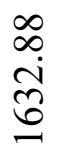 & $\begin{array}{l}\text { テे } \\
\stackrel{0}{n} \\
\end{array}$ & $\begin{array}{l}n \\
\infty \\
0 \\
0 \\
=\end{array}$ & 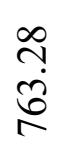 & $\begin{array}{c}\tilde{n} \\
\stackrel{\Im}{0}\end{array}$ & $\begin{array}{l}\vec{\nabla} \\
\dot{0} \\
i n \\
i n\end{array}$ & 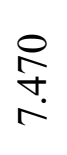 & $\underset{\infty}{\stackrel{I}{ \pm}}$ & $\begin{array}{l}\text { ㄱ. } \\
\stackrel{0}{0}\end{array}$ & 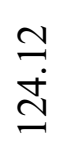 & $\begin{array}{l} \pm \\
\stackrel{0}{0} \\
\pm\end{array}$ \\
\hline$N$ & $\stackrel{\overline{0}}{\stackrel{1}{\Xi}}$ & 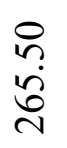 & $\begin{array}{l}\overrightarrow{7} \\
6 \\
6\end{array}$ & 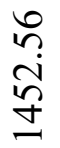 & $\begin{array}{l}\stackrel{8}{0} \\
\text { ㅁ } \\
\text { I }\end{array}$ & $\begin{array}{c}\infty \\
\stackrel{n}{\infty} \\
\infty\end{array}$ & $\begin{array}{l}\stackrel{a}{+} \\
\infty \\
\stackrel{0}{0} \\
0\end{array}$ & 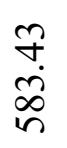 & $\underset{\infty}{\stackrel{m}{0}}$ & 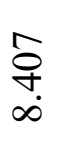 & \begin{tabular}{l}
\multirow{\sigma}{*}{} \\
$\stackrel{2}{a}$
\end{tabular} & $\begin{array}{l} \pm \\
\stackrel{\Xi}{二}\end{array}$ & 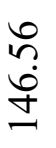 \\
\hline$m$ & 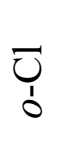 & $\begin{array}{l}\stackrel{n}{n} \\
\ddot{n} \\
\text { in }\end{array}$ & 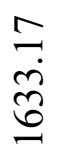 & $\begin{array}{l}\stackrel{8}{\Omega} \\
\stackrel{2}{\Omega} \\
\end{array}$ & \begin{tabular}{l}
\multirow{n}{*}{} \\
$\stackrel{n}{\Xi}$ \\
$=$
\end{tabular} & $\begin{array}{l}\stackrel{ }{\longrightarrow} \\
\stackrel{\leftrightarrow}{+}\end{array}$ & 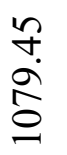 & $\frac{n}{\stackrel{n}{n}}$ & $\begin{array}{l}\stackrel{\circ}{7} \\
\stackrel{r}{r}\end{array}$ & $\underset{\infty}{\stackrel{\Xi}{\infty}}$ & \begin{tabular}{l}
\multirow{2}{*}{} \\
$\stackrel{2}{2}$ \\
\end{tabular} & 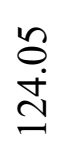 & 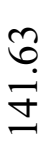 \\
\hline$\nabla$ & $\begin{array}{l}\underbrace{}_{1} \\
\vdots\end{array}$ & $\begin{array}{l}\stackrel{i}{n} \\
\underset{\infty}{\infty} \\
\text { N }\end{array}$ & $\begin{array}{l}8 \\
8 \\
8 \\
8\end{array}$ & $\begin{array}{l}\infty \\
0 \\
0 \\
\tilde{n} \\
n\end{array}$ & $\begin{array}{l}\text { ণે } \\
\text { ஸ̃ } \\
\text { = }\end{array}$ & $\frac{\vec{b}}{\dot{0}}$ & $\begin{array}{l}\stackrel{N}{a} \\
a \\
\sigma\end{array}$ & 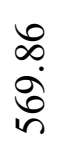 & $\underset{r}{\stackrel{\overbrace{}}{\sim}}$ & 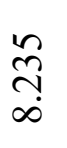 & $\begin{array}{l}\vec{\nabla} \\
\stackrel{2}{\sigma}\end{array}$ & $\stackrel{\stackrel{\sim}{ \pm}}{\stackrel{ \pm}{\beth}}$ & $\begin{array}{l}\infty \\
0 \\
\stackrel{\Xi}{ \pm}\end{array}$ \\
\hline$n$ & $\begin{array}{l}U_{1} \\
2\end{array}$ & $\begin{array}{l}\stackrel{0}{n} \\
\text { ¿্ঠ }\end{array}$ & \begin{tabular}{l}
$\infty$ \\
0 \\
$\ddot{1}$ \\
$\tilde{n}$ \\
\hdashline
\end{tabular} & 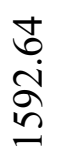 & $\begin{array}{l}\hat{0} \\
\stackrel{0}{\Xi} \\
\equiv\end{array}$ & $\begin{array}{l}\stackrel{2}{1} \\
\stackrel{0}{\infty} \\
\stackrel{0}{r}\end{array}$ & $\begin{array}{l}\frac{1}{n} \\
\text { ñ } \\
0\end{array}$ & $\begin{array}{l}\text { J } \\
\text { î } \\
\text { \&े }\end{array}$ & $\underset{\circ}{\stackrel{D}{0}}$ & $\begin{array}{l}\bullet \\
\infty \\
0 \\
\end{array}$ & $\begin{array}{l}\tilde{b} \\
\mathfrak{2} \\
2\end{array}$ & $\begin{array}{l}\underset{+}{+} \\
\stackrel{+}{\beth}\end{array}$ & $\begin{array}{l}\dot{J} \\
\dot{J}\end{array}$ \\
\hline 6 & $\frac{1}{1}$ & $\begin{array}{l}\stackrel{8}{0} \\
\stackrel{+}{\sim}\end{array}$ & $\begin{array}{l}8 \\
8 \\
\infty \\
0 \\
0 \\
0\end{array}$ & 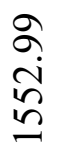 & \begin{tabular}{l}
$\simeq$ \\
\multirow{6}{\sigma}{} \\
$=$
\end{tabular} & $\begin{array}{l}\text { oे } \\
\text { i. } \\
\stackrel{0}{r}\end{array}$ & $\begin{array}{l}\text { ñ } \\
\infty \\
0 \\
0\end{array}$ & $\begin{array}{l}2 \\
\stackrel{2}{0} \\
\frac{\pi}{6}\end{array}$ & ڤั & $\stackrel{5}{\stackrel{2}{r}}$ & $\begin{array}{l}\bar{n} \\
\infty \\
\infty\end{array}$ & 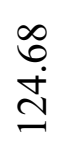 & 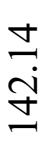 \\
\hline$r$ & $\underbrace{m}_{1}$ & $\begin{array}{l}\stackrel{i}{n} \\
\stackrel{+}{N}\end{array}$ & $\begin{array}{l}\stackrel{a}{7} \\
\dot{0} \\
\stackrel{0}{-}\end{array}$ & $\begin{array}{l}\bar{n} \\
\infty \\
\infty \\
n\end{array}$ & $\begin{array}{l}\underset{b}{0} \\
\dot{\sigma} \\
=\end{array}$ & \begin{tabular}{l}
\multirow{2}{*}{} \\
రे \\
$\infty$
\end{tabular} & $\begin{array}{l}\bar{\sigma} \\
\text { ஸे } \\
\text { ô }\end{array}$ & $\begin{array}{l}0 \\
\infty \\
0 \\
0 \\
0\end{array}$ & $\underset{\sim}{\stackrel{\infty}{\sim}}$ & $\begin{array}{l}0 \\
\stackrel{0}{0} \\
\stackrel{0}{r}\end{array}$ & 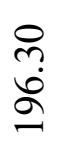 & $\stackrel{\stackrel{\sim}{\beth}}{\stackrel{ \pm}{\beth}}$ & 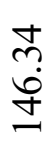 \\
\hline$\infty$ & $\begin{array}{c}\delta^{N} \\
Z_{1} \\
\vdots\end{array}$ & $\begin{array}{l}\stackrel{n}{n} \\
\ddot{b} \\
\stackrel{n}{n}\end{array}$ & 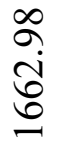 & $\begin{array}{l}\overline{0} \\
\text { ลे } \\
\text { กิ }\end{array}$ & $\begin{array}{l}\mathfrak{Z} \\
\text { ป } \\
\text { ב }\end{array}$ & $\underset{\infty}{\stackrel{\infty}{N}}$ & 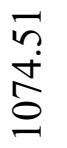 & $\begin{array}{l}0 \\
0 \\
\ddot{n} \\
i n\end{array}$ & $\underset{\sim}{\mathbb{N}}$ & $\frac{N}{\infty}$ & 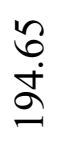 & $\begin{array}{l}\infty \\
0 \\
\mathbb{I} \\
\text { I }\end{array}$ & $\begin{array}{l}\stackrel{n}{\sim} \\
\stackrel{\infty}{+}\end{array}$ \\
\hline$a$ & $\begin{array}{l}O_{1}^{2} \\
Z_{1}\end{array}$ & $\begin{array}{l}\stackrel{n}{n} \\
\stackrel{n}{n} \\
\end{array}$ & 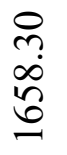 & $\begin{array}{l}n \\
n \\
\dot{n} \\
n \\
n\end{array}$ & $\begin{array}{l}\Omega \\
\Xi \\
\Xi\end{array}$ & $\begin{array}{l}\infty \\
0 \\
\infty \\
\stackrel{\infty}{人}\end{array}$ & $\stackrel{\widetilde{N}}{\stackrel{ }{0}}$ & $\begin{array}{l}\stackrel{0}{\circ} \\
\stackrel{0}{8}\end{array}$ & $\frac{n}{\sigma}$ & $\underset{\infty}{\stackrel{n}{\sim}}$ & $\begin{array}{l}\stackrel{n}{n} \\
\mathfrak{n} \\
\stackrel{n}{n}\end{array}$ & $\stackrel{m}{\stackrel{m}{ \pm}}$ & 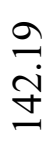 \\
\hline
\end{tabular}


Table 3. Results of statistical analysis of ultraviolet absorption maxima ( $\lambda$ max, $n m)$, infrared absorptions ( $v$, $\left.\mathrm{cm}^{-1}\right)$ and NMR chemical shifts $(\delta, \mathrm{ppm})$ of substituted styryl 1-pyrenyl ketones with Hammett $\sigma, \sigma^{+}, \sigma_{\mathrm{I}}, \sigma_{\mathrm{R}}$ and $\mathrm{F}$ and $\mathrm{R}$ parameters.

\begin{tabular}{|c|c|c|c|c|c|c|c|}
\hline Frequency & Constants & $\mathbf{r}$ & I & $\boldsymbol{\rho}$ & $\mathbf{S}$ & $\mathbf{n}$ & $\begin{array}{l}\text { Correlated } \\
\text { derivatives }\end{array}$ \\
\hline \multirow[t]{6}{*}{$\mathbf{U V} \lambda_{\max }$} & $\sigma$ & 0.700 & 274.53 & 0.271 & 18.15 & 9 & $\begin{array}{l}\mathrm{H}, 3-\mathrm{Br}, 2-\mathrm{Cl}, 3-\mathrm{Cl}, 4-\mathrm{Cl}, \\
4-\mathrm{F}, 4-\mathrm{CH}_{3}, 3-\mathrm{NO}_{2}, 4-\mathrm{NO}_{2}\end{array}$ \\
\hline & $\sigma^{+}$ & 0.707 & 273.59 & 3.658 & 18.09 & 9 & $\begin{array}{l}\mathrm{H}, 3-\mathrm{Br}, 2-\mathrm{Cl}, 3-\mathrm{Cl}, 4-\mathrm{Cl}, \\
4-\mathrm{F}, 4-\mathrm{CH}_{3}, 3-\mathrm{NO}_{2}, 4-\mathrm{NO}_{2}\end{array}$ \\
\hline & $\sigma_{\mathrm{I}}$ & 0.813 & 278.35 & -9.200 & 17.97 & 9 & $\begin{array}{l}\mathrm{H}, 3-\mathrm{Br}, 2-\mathrm{Cl}, 3-\mathrm{Cl}, 4-\mathrm{Cl}, \\
4-\mathrm{F}, 4-\mathrm{CH}_{3}, 3-\mathrm{NO}_{2}, 4-\mathrm{NO}_{2}\end{array}$ \\
\hline & $\sigma_{\mathrm{R}}$ & 0.823 & 276.93 & 19.938 & 17.65 & 9 & $\begin{array}{l}\mathrm{H}, 3-\mathrm{Br}, 2-\mathrm{Cl}, 3-\mathrm{Cl}, 4-\mathrm{Cl}, \\
4-\mathrm{F}, 4-\mathrm{CH}_{3}, 3-\mathrm{NO}_{2}, 4-\mathrm{NO}_{2}\end{array}$ \\
\hline & $\mathrm{F}$ & 0.936 & 284.52 & -23.733 & 16.88 & 7 & $\begin{array}{l}\text { 3-Br, 2-Cl, 3-Cl, 4-Cl, } \\
\text { 4-F, 3- } \mathrm{NO}_{2}, 4-\mathrm{NO}_{2}\end{array}$ \\
\hline & $\mathrm{R}$ & 0.735 & 278.70 & 27.291 & 16.94 & 9 & $\begin{array}{l}\mathrm{H}, 3-\mathrm{Br}, 2-\mathrm{Cl}, 3-\mathrm{Cl}, 4-\mathrm{Cl}, \\
\text { 4-F, 4- } \mathrm{CH}_{3}, 3-\mathrm{NO}_{2}, 4-\mathrm{NO}_{2}\end{array}$ \\
\hline \multirow[t]{6}{*}{$v \mathrm{CO}_{\text {s-cis }}\left(\mathrm{cm}^{-1}\right)$} & $\sigma$ & 0.823 & 1634.82 & 26.691 & 26.04 & 9 & $\begin{array}{l}\mathrm{H}, 3-\mathrm{Br}, 2-\mathrm{Cl}, 3-\mathrm{Cl}, 4-\mathrm{Cl}, \\
\text { 4-F, 4- } \mathrm{CH}_{3}, 3-\mathrm{NO}_{2}, 4-\mathrm{NO}_{2}\end{array}$ \\
\hline & $\sigma^{+}$ & 0.726 & 1637.42 & 18.291 & 26.63 & 9 & $\begin{array}{l}\mathrm{H}, 3-\mathrm{Br}, 2-\mathrm{Cl}, 3-\mathrm{Cl}, 4-\mathrm{Cl}, \\
\text { 4-F, 4-- } \mathrm{CH}_{3}, 3-\mathrm{NO}_{2}, 4-\mathrm{NO}_{2}\end{array}$ \\
\hline & $\sigma_{\mathrm{I}}$ & 0.510 & 1640.39 & 5.195 & 27.56 & 9 & $\begin{array}{l}\mathrm{H}, 3-\mathrm{Br}, 2-\mathrm{Cl}, 3-\mathrm{Cl}, 4-\mathrm{Cl}, \\
4-\mathrm{F}, 4-\mathrm{CH}_{3}, 3-\mathrm{NO}_{2}, 4-\mathrm{NO}_{2}\end{array}$ \\
\hline & $\sigma_{\mathrm{R}}$ & 0.905 & 1651.07 & 73.370 & 22.80 & 9 & $\begin{array}{l}\mathrm{H}, 3-\mathrm{Br}, 2-\mathrm{Cl}, 3-\mathrm{Cl}, 4-\mathrm{Cl}, \\
\text { 4-F, 4- } \mathrm{CH}_{3}, 3-\mathrm{NO}_{2}, 4-\mathrm{NO}_{2}\end{array}$ \\
\hline & $\mathrm{F}$ & 0.848 & 1644.50 & -4.766 & 27.57 & 9 & $\begin{array}{l}\mathrm{H}, 3-\mathrm{Br}, 2-\mathrm{Cl}, 3-\mathrm{Cl}, 4-\mathrm{Cl}, \\
\text { 4-F, 4-- } \mathrm{CH}_{3}, 3-\mathrm{NO}_{2}, 4-\mathrm{NO}_{2}\end{array}$ \\
\hline & $\mathrm{R}$ & 0.905 & 1651.33 & 58.800 & 23.78 & 7 & $\begin{array}{c}\mathrm{H}, 2-\mathrm{Cl}, 4-\mathrm{Cl}, 4-\mathrm{F}, 4-\mathrm{CH}_{3}, \\
3-\mathrm{NO}_{2}, 4-\mathrm{NO}_{2}\end{array}$ \\
\hline \multirow[t]{6}{*}{$v \mathrm{CO}_{\text {s-trans }}\left(\mathrm{cm}^{-1}\right)$} & $\sigma$ & 0.929 & 1573.48 & -43.224 & 48.57 & 7 & $\begin{array}{c}\mathrm{H}, 2-\mathrm{Cl}, 3-\mathrm{Cl}, 4-\mathrm{Cl}, 4-\mathrm{F} \\
\text { 4- } \mathrm{CH}_{3}, 3-\mathrm{NO}_{2}\end{array}$ \\
\hline & $\sigma^{+}$ & 0.825 & 1570.22 & -32.992 & 49.06 & 9 & $\begin{array}{l}\mathrm{H}, 3-\mathrm{Br}, 2-\mathrm{Cl}, 3-\mathrm{Cl}, 4-\mathrm{Cl}, \\
\text { 4-F, 4-- } \mathrm{CH}_{3}, 3-\mathrm{NO}_{2}, 4-\mathrm{NO}_{2}\end{array}$ \\
\hline & $\sigma_{\mathrm{I}}$ & 0.820 & 1586.07 & -61.563 & 47.95 & 9 & $\begin{array}{l}\mathrm{H}, 3-\mathrm{Br}, 2-\mathrm{Cl}, 3-\mathrm{Cl}, 4-\mathrm{Cl}, \\
\text { 4-F, 4- } \mathrm{CH}_{3}, 3-\mathrm{NO}_{2}, 4-\mathrm{NO}_{2}\end{array}$ \\
\hline & $\sigma_{\mathrm{R}}$ & 0.770 & 1563.24 & 18.929 & 50.62 & 9 & $\begin{array}{l}\mathrm{H}, 3-\mathrm{Br}, 2-\mathrm{Cl}, 3-\mathrm{Cl}, 4-\mathrm{Cl}, \\
\text { 4-F, 4- } \mathrm{CH}_{3}, 3-\mathrm{NO}_{2}, 4-\mathrm{NO}_{2}\end{array}$ \\
\hline & $\mathrm{F}$ & 0.932 & 1585.23 & -57.920 & 48.09 & 8 & $\begin{array}{c}\mathrm{H}, 2-\mathrm{Cl}, 3-\mathrm{Cl}, 4-\mathrm{Cl}, 4-\mathrm{F} \\
\text { 4- } \mathrm{CH}_{3}, 3-\mathrm{NO}_{2}, 4-\mathrm{NO}_{2}\end{array}$ \\
\hline & $\mathrm{R}$ & 0.815 & 1565.90 & 32.463 & 50.18 & 9 & $\begin{array}{l}\mathrm{H}, 3-\mathrm{Br}, 2-\mathrm{Cl}, 3-\mathrm{Cl}, 4-\mathrm{Cl}, \\
\text { 4-F, 4- } \mathrm{CH}_{3}, 3-\mathrm{NO}_{2}, 4-\mathrm{NO}_{2}\end{array}$ \\
\hline \multirow[t]{4}{*}{$v C H_{i p}\left(\mathrm{~cm}^{-1}\right)$} & $\sigma$ & 0.731 & 1141.58 & -24.769 & 25.43 & 9 & $\begin{array}{l}\mathrm{H}, 3-\mathrm{Br}, 2-\mathrm{Cl}, 3-\mathrm{Cl}, 4-\mathrm{Cl}, \\
4-\mathrm{F}, 4-\mathrm{CH}_{3}, 3-\mathrm{NO}_{2}, 4-\mathrm{NO}_{2}\end{array}$ \\
\hline & $\sigma^{+}$ & 0.746 & 1142.11 & -27.509 & 24.50 & 9 & $\begin{array}{l}\mathrm{H}, 3-\mathrm{Br}, 2-\mathrm{Cl}, 3-\mathrm{Cl}, 4-\mathrm{Cl}, \\
\text { 4-F, 4-- } \mathrm{CH}_{3}, 3-\mathrm{NO}_{2}, 4-\mathrm{NO}_{2}\end{array}$ \\
\hline & $\sigma_{\mathrm{I}}$ & 0.696 & 1137.25 & -6.887 & 26.75 & 9 & $\begin{array}{l}\mathrm{H}, 3-\mathrm{Br}, 2-\mathrm{Cl}, 3-\mathrm{Cl}, 4-\mathrm{Cl}, \\
\text { 4-F, 4- } \mathrm{CH}_{3}, 3-\mathrm{NO}_{2}, 4-\mathrm{NO}_{2}\end{array}$ \\
\hline & $\sigma_{\mathrm{R}}$ & 0.950 & 1126.95 & -64.242 & 23.09 & 8 & $\begin{array}{c}\mathrm{H}, 3-\mathrm{Br}, 3-\mathrm{Cl}, 4-\mathrm{Cl}, 4-\mathrm{F} \\
4-\mathrm{CH}_{3}, 3-\mathrm{NO}_{2}, 4-\mathrm{NO}_{2}\end{array}$ \\
\hline
\end{tabular}




\begin{tabular}{|c|c|c|c|c|c|c|c|}
\hline & $\mathrm{F}$ & 0.710 & 1130.38 & 9.739 & 26.67 & 9 & $\begin{array}{c}\mathrm{H}, 2-\mathrm{Cl}, 3-\mathrm{Cl}, 4-\mathrm{Cl}, 4-\mathrm{F} \\
4-\mathrm{CH}_{3}, 3-\mathrm{NO}_{2}, 4-\mathrm{NO}_{2}\end{array}$ \\
\hline & $\mathrm{R}$ & 0.906 & 1123.81 & -70.923 & 20.82 & 9 & $\begin{array}{l}\mathrm{H}, 3-\mathrm{Br}, 2-\mathrm{Cl}, 3-\mathrm{Cl}, 4-\mathrm{Cl}, \\
4-\mathrm{F}, 4-\mathrm{CH}_{3}, 3-\mathrm{NO}_{2}, 4-\mathrm{NO}_{2}\end{array}$ \\
\hline \multirow[t]{6}{*}{$v \mathrm{CH}_{o p}\left(\mathrm{~cm}^{-1}\right)$} & $\sigma$ & 0.904 & 802.65 & -61.741 & 47.33 & 8 & $\begin{array}{c}\mathrm{H}, 2-\mathrm{Cl}, 3-\mathrm{Cl}, 4-\mathrm{Cl}, 4-\mathrm{F} \\
\text { 4- } \mathrm{CH}_{3}, 3-\mathrm{NO}_{2}, 4-\mathrm{NO}_{2}\end{array}$ \\
\hline & $\sigma^{+}$ & 0.849 & 802.83 & -64.510 & 45.10 & 8 & $\begin{array}{c}\mathrm{H}, 2-\mathrm{Cl}, 3-\mathrm{Cl}, 4-\mathrm{Cl}, 4-\mathrm{F} \\
4-\mathrm{CH}_{3}, 3-\mathrm{NO}_{2}, 4-\mathrm{NO}_{2}\end{array}$ \\
\hline & $\sigma_{\mathrm{I}}$ & 0.744 & 819.31 & -84.663 & 46.48 & 9 & $\begin{array}{l}\mathrm{H}, 3-\mathrm{Br}, 2-\mathrm{Cl}, 3-\mathrm{Cl}, 4-\mathrm{Cl}, \\
\text { 4-F, 4- } \mathrm{CH}_{3}, 3-\mathrm{NO}_{2}, 4-\mathrm{NO}_{2}\end{array}$ \\
\hline & $\sigma_{\mathrm{R}}$ & 0.718 & 779.62 & -45.019 & 50.95 & 9 & $\begin{array}{c}\mathrm{H}, 3-\mathrm{Br}, 3-\mathrm{Cl}, 4-\mathrm{Cl}, 4-\mathrm{F} \\
\text { 4- } \mathrm{CH}_{3}, 3-\mathrm{NO}_{2}, 4-\mathrm{NO}_{2}\end{array}$ \\
\hline & $\mathrm{F}$ & 0.737 & 813.91 & -69.487 & 48.02 & 9 & $\begin{array}{c}\mathrm{H}, 2-\mathrm{Cl}, 3-\mathrm{Cl}, 4-\mathrm{Cl}, 4-\mathrm{F} \\
\text { 4- } \mathrm{CH}_{3}, 3-\mathrm{NO}_{2}, 4-\mathrm{NO}_{2}\end{array}$ \\
\hline & $\mathrm{R}$ & 0.727 & 775.77 & -60.733 & 49.78 & 9 & $\begin{array}{l}\mathrm{H}, 3-\mathrm{Br}, 2-\mathrm{Cl}, 3-\mathrm{Cl}, 4-\mathrm{Cl}, \\
\text { 4-F, 4-- } \mathrm{CH}_{3}, 3-\mathrm{NO}_{2}, 4-\mathrm{NO}_{2}\end{array}$ \\
\hline \multirow[t]{6}{*}{$v \mathrm{CH}=\mathrm{CH}_{o p}\left(\mathrm{~cm}^{-1}\right)$} & $\sigma$ & 0.945 & 1044.73 & 38.255 & 25.93 & 8 & $\begin{array}{c}\mathrm{H}, 3-\mathrm{Br}, 2-\mathrm{Cl}, 4-\mathrm{Cl}, 4-\mathrm{F} \\
\text { 4- } \mathrm{CH}_{3}, 3-\mathrm{NO}_{2}, 4-\mathrm{NO}_{2}\end{array}$ \\
\hline & $\sigma^{+}$ & 0.954 & 1044.59 & 40.063 & 24.33 & 8 & $\begin{array}{c}\mathrm{H}, 3-\mathrm{Br}, 2-\mathrm{Cl}, 4-\mathrm{Cl}, 4-\mathrm{F} \\
\text { 4- } \mathrm{CH}_{3}, 3-\mathrm{NO}_{2}, 4-\mathrm{NO}_{2}\end{array}$ \\
\hline & $\sigma_{\mathrm{I}}$ & 0.725 & 1044.71 & 27.145 & 28.11 & 9 & $\begin{array}{l}\mathrm{H}, 3-\mathrm{Br}, 2-\mathrm{Cl}, 3-\mathrm{Cl}, 4-\mathrm{Cl}, \\
4-\mathrm{F}, 4-\mathrm{CH}_{3}, 3-\mathrm{NO}_{2}, 4-\mathrm{NO}_{2}\end{array}$ \\
\hline & $\sigma_{\mathrm{R}}$ & 0.751 & 1063.98 & 70.592 & 24.90 & 9 & $\begin{array}{c}\mathrm{H}, 3-\mathrm{Br}, 3-\mathrm{Cl}, 4-\mathrm{Cl}, 4-\mathrm{F} \\
\text { 4- } \mathrm{CH}_{3}, 3-\mathrm{NO}_{2}, 4-\mathrm{NO}_{2}\end{array}$ \\
\hline & $\mathrm{F}$ & 0.707 & 1052.72 & 7.250 & 28.98 & 9 & $\begin{array}{c}\mathrm{H}, 2-\mathrm{Cl}, 3-\mathrm{Cl}, 4-\mathrm{Cl}, 4-\mathrm{F}, \\
\text { 4- } \mathrm{CH}_{3}, 3-\mathrm{NO}_{2}, 4-\mathrm{NO}_{2}\end{array}$ \\
\hline & $\mathrm{R}$ & 0.858 & 1066.38 & 70.903 & 23.64 & 9 & $\begin{array}{l}\mathrm{H}, 3-\mathrm{Br}, 2-\mathrm{Cl}, 3-\mathrm{Cl}, 4-\mathrm{Cl}, \\
\text { 4-F, 4-- } \mathrm{CH}_{3}, 3-\mathrm{NO}_{2}, 4-\mathrm{NO}_{2}\end{array}$ \\
\hline \multirow[t]{6}{*}{$v C=C_{o p}\left(\mathrm{~cm}^{-1}\right)$} & $\sigma$ & 0.927 & 592.25 & -17.959 & 21.69 & 7 & $\begin{array}{c}\text { 3- } \mathrm{Br}, 2-\mathrm{Cl}, 3-\mathrm{Cl}, 4-\mathrm{Cl}, \\
\text { 4-F, 4-CH }, 3-\mathrm{NO}_{2}\end{array}$ \\
\hline & $\sigma^{+}$ & 0.935 & 592.68 & -20.090 & 21.09 & 7 & $\begin{array}{l}\text { 3-Br, 2-Cl, 3-Cl, 4-Cl, } \\
\text { 4-F, 4- } \mathrm{CH}_{3}, 3-\mathrm{NO}_{2}\end{array}$ \\
\hline & $\sigma_{\mathrm{I}}$ & 0.708 & 584.21 & 7.057 & 22.46 & 9 & $\begin{array}{l}\mathrm{H}, 3-\mathrm{Br}, 2-\mathrm{Cl}, 3-\mathrm{Cl}, 4-\mathrm{Cl}, \\
4-\mathrm{F}, 4-\mathrm{CH}_{3}, 3-\mathrm{NO}_{2}, 4-\mathrm{NO}_{2}\end{array}$ \\
\hline & $\sigma_{\mathrm{R}}$ & 0.950 & 580.79 & -53.970 & 19.43 & 7 & $\begin{array}{c}\mathrm{H}, 3-\mathrm{Br}, 2-\mathrm{Cl}, 3-\mathrm{Cl}, 4-\mathrm{Cl}, \\
\text { 4-F, 3-NO }\end{array}$ \\
\hline & $\mathrm{F}$ & 0.821 & 579.73 & 17.589 & 22.00 & 9 & $\begin{array}{c}\mathrm{H}, 2-\mathrm{Cl}, 3-\mathrm{Cl}, 4-\mathrm{Cl}, 4-\mathrm{F} \\
\text { 4-CH } \\
\end{array}$ \\
\hline & $\mathrm{R}$ & 0.855 & 579.21 & -52.486 & 18.76 & 9 & $\begin{array}{l}\mathrm{H}, 3-\mathrm{Br}, 2-\mathrm{Cl}, 3-\mathrm{Cl}, 4-\mathrm{Cl}, \\
\text { 4-F, 4-- } \mathrm{CH}_{3}, 3-\mathrm{NO}_{2}, 4-\mathrm{NO}_{2}\end{array}$ \\
\hline \multirow[t]{5}{*}{$\delta_{\mathrm{H \alpha}}(\mathrm{ppm})$} & $\sigma$ & 0.955 & 7.333 & 0.446 & 0.30 & 6 & $\begin{array}{c}\mathrm{H}, 2-\mathrm{Cl}, 3-\mathrm{Cl}, 4-\mathrm{CH}_{3}, \\
\text { 3-NO } \mathrm{NO}_{2}, 4-\mathrm{NO}_{2}\end{array}$ \\
\hline & $\sigma^{+}$ & 0.957 & 7.338 & 0.442 & 0.29 & 6 & $\begin{array}{c}\mathrm{H}, 2-\mathrm{Cl}, 3-\mathrm{Cl}, 4-\mathrm{CH}_{3}, \\
\text { 3- } \mathrm{NO}_{2}, 4-\mathrm{NO}_{2}\end{array}$ \\
\hline & $\sigma_{\mathrm{I}}$ & 0.718 & 7.424 & 0.090 & 0.34 & 9 & $\begin{array}{l}\mathrm{H}, 3-\mathrm{Br}, 2-\mathrm{Cl}, 3-\mathrm{Cl}, 4-\mathrm{Cl}, \\
4-\mathrm{F}, 4-\mathrm{CH}_{3}, 3-\mathrm{NO}_{2}, 4-\mathrm{NO}_{2}\end{array}$ \\
\hline & $\sigma_{\mathrm{R}}$ & 0.951 & 7.558 & 0.829 & 0.29 & 7 & $\begin{array}{c}\mathrm{H}, 3-\mathrm{Br}, 2-\mathrm{Cl}, 3-\mathrm{Cl}, 4-\mathrm{Cl}, \\
\text { 4-F, 3-NO }\end{array}$ \\
\hline & $\mathrm{F}$ & 0.645 & 7.494 & -0.078 & 0.34 & 8 & $\begin{array}{c}\mathrm{H}, 2-\mathrm{Cl}, 3-\mathrm{Cl}, 4-\mathrm{Cl}, 4-\mathrm{F} \\
4-\mathrm{CH}_{3}, 3-\mathrm{NO}_{2}, 4-\mathrm{NO}_{2}\end{array}$ \\
\hline
\end{tabular}




\begin{tabular}{|c|c|c|c|c|c|c|c|}
\hline & $\mathrm{R}$ & 0.989 & 7.565 & 0.692 & 0.30 & 7 & $\begin{array}{c}\mathrm{H}, 2-\mathrm{Cl}, 3-\mathrm{Cl}, 4-\mathrm{F}, 4-\mathrm{CH}_{3}, \\
\text { 3-NO } \mathrm{NO}_{2}, 4-\mathrm{NO}_{2}\end{array}$ \\
\hline \multirow[t]{6}{*}{$\delta_{\mathrm{H} \beta}(\mathbf{p p m})$} & $\sigma$ & 0.960 & 7.880 & 0.551 & 0.24 & 7 & $\begin{array}{c}\mathrm{H}, 3-\mathrm{Br}, 2-\mathrm{Cl}, 3-\mathrm{Cl}, 4-\mathrm{Cl}, \\
\text { 4-F, 4-CH3 }\end{array}$ \\
\hline & $\sigma^{+}$ & 0.974 & 7.875 & 0.587 & 0.20 & 7 & $\begin{array}{c}\mathrm{H}, 3-\mathrm{Br}, 2-\mathrm{Cl}, 3-\mathrm{Cl}, 4-\mathrm{Cl} \\
\text { 4-F, 4- } \mathrm{CH}_{3}\end{array}$ \\
\hline & $\sigma_{\mathrm{I}}$ & 0.834 & 7.877 & 0.398 & 0.29 & 9 & $\begin{array}{l}\mathrm{H}, 3-\mathrm{Br}, 2-\mathrm{Cl}, 3-\mathrm{Cl}, 4-\mathrm{Cl}, \\
4-\mathrm{F}, 4-\mathrm{CH}_{3}, 3-\mathrm{NO}_{2}, 4-\mathrm{NO}_{2}\end{array}$ \\
\hline & $\sigma_{\mathrm{R}}$ & 0.740 & 8.108 & 0.592 & 0.28 & 9 & $\begin{array}{l}\mathrm{H}, 3-\mathrm{Br}, 2-\mathrm{Cl}, 3-\mathrm{Cl}, 4-\mathrm{Cl}, \\
\text { 4-F, 4-- } \mathrm{CH}_{3}, 3-\mathrm{NO}_{2}, 4-\mathrm{NO}_{2}\end{array}$ \\
\hline & $\mathrm{F}$ & 0.717 & 7.957 & 0.195 & 0.30 & 9 & $\begin{array}{l}\mathrm{H}, 3-\mathrm{Br}, 2-\mathrm{Cl}, 3-\mathrm{Cl}, 4-\mathrm{Cl}, \\
4-\mathrm{F}, 4-\mathrm{CH}_{3}, 3-\mathrm{NO}_{2}, 4-\mathrm{NO}_{2}\end{array}$ \\
\hline & $\mathrm{R}$ & 0.844 & 8.125 & 0.578 & 0.28 & 9 & $\begin{array}{l}\mathrm{H}, 3-\mathrm{Br}, 2-\mathrm{Cl}, 3-\mathrm{Cl}, 4-\mathrm{Cl}, \\
4-\mathrm{F}, 4-\mathrm{CH}_{3}, 3-\mathrm{NO}_{2}, 4-\mathrm{NO}_{2}\end{array}$ \\
\hline \multirow[t]{6}{*}{$\delta \mathrm{CO}(\mathrm{ppm})$} & $\sigma$ & 0.991 & 194.60 & 0.847 & 2.57 & 8 & $\begin{array}{c}\mathrm{H}, 3-\mathrm{Br}, 2-\mathrm{Cl}, 3-\mathrm{Cl}, 4-\mathrm{Cl} \\
4-\mathrm{CH}_{3}, 3-\mathrm{NO}_{2}, 4-\mathrm{NO}_{2}\end{array}$ \\
\hline & $\sigma^{+}$ & 0.919 & 194.48 & 1.304 & 2.53 & 8 & $\begin{array}{c}\mathrm{H}, 3-\mathrm{Br}, 2-\mathrm{Cl}, 3-\mathrm{Cl}, 4-\mathrm{Cl}, \\
\text { 4- } \mathrm{CH}_{3}, 3-\mathrm{NO}_{2}, 4-\mathrm{NO}_{2}\end{array}$ \\
\hline & $\sigma_{\mathrm{I}}$ & 0.934 & 196.20 & -3.330 & 2.42 & 8 & $\begin{array}{c}\mathrm{H}, 3-\mathrm{Br}, 2-\mathrm{Cl}, 3-\mathrm{Cl}, 4-\mathrm{Cl} \\
\text { 4- } \mathrm{CH}_{3}, 3-\mathrm{NO}_{2}, 4-\mathrm{NO}_{2}\end{array}$ \\
\hline & $\sigma_{\mathrm{R}}$ & 0.861 & 195.72 & 7.518 & 2.04 & 9 & $\begin{array}{l}\mathrm{H}, 3-\mathrm{Br}, 2-\mathrm{Cl}, 3-\mathrm{Cl}, 4-\mathrm{Cl}, \\
\text { 4-F, 4-- } \mathrm{CH}_{3}, 3-\mathrm{NO}_{2}, 4-\mathrm{NO}_{2}\end{array}$ \\
\hline & $\mathrm{F}$ & 0.959 & 197.12 & -5.456 & 2.08 & 8 & $\begin{array}{c}\mathrm{H}, 3-\mathrm{Br}, 2-\mathrm{Cl}, 3-\mathrm{Cl}, 4-\mathrm{Cl} \\
4-\mathrm{CH}_{3}, 3-\mathrm{NO}_{2}, 4-\mathrm{NO}_{2}\end{array}$ \\
\hline & $\mathrm{R}$ & 0.865 & 195.95 & 7.402 & 1.89 & 9 & $\begin{array}{l}\mathrm{H}, 3-\mathrm{Br}, 2-\mathrm{Cl}, 3-\mathrm{Cl}, 4-\mathrm{Cl}, \\
\text { 4-F, 4-- } \mathrm{CH}_{3}, 3-\mathrm{NO}_{2}, 4-\mathrm{NO}_{2}\end{array}$ \\
\hline \multirow[t]{6}{*}{$\delta \mathrm{C} \alpha(\mathrm{ppm})$} & $\sigma$ & 0.737 & 124.04 & -1.283 & 1.07 & 9 & $\begin{array}{c}\mathrm{H}, 3-\mathrm{Br}, 2-\mathrm{Cl}, 3-\mathrm{Cl}, 4-\mathrm{Cl}, \\
\text { 4- } \mathrm{CH}_{3}, 3-\mathrm{NO}_{2}, 4-\mathrm{NO}_{2}\end{array}$ \\
\hline & $\sigma^{+}$ & 0.837 & 123.98 & -1.106 & 1.07 & 9 & $\begin{array}{c}\mathrm{H}, 3-\mathrm{Br}, 2-\mathrm{Cl}, 3-\mathrm{Cl}, 4-\mathrm{Cl}, \\
\text { 4- } \mathrm{CH}_{3}, 3-\mathrm{NO}_{2}, 4-\mathrm{NO}_{2}\end{array}$ \\
\hline & $\sigma_{\mathrm{I}}$ & 0.952 & 124.04 & -0.914 & 1.13 & 7 & $\begin{array}{c}\mathrm{H}, 3-\mathrm{Br}, 2-\mathrm{Cl}, 4-\mathrm{Cl}, 4-\mathrm{F}, \\
\text { 3- } \mathrm{NO}_{2}, 4-\mathrm{NO}_{2}\end{array}$ \\
\hline & $\sigma_{\mathrm{R}}$ & 0.727 & 123.50 & -1.496 & 1.12 & 9 & $\begin{array}{l}\mathrm{H}, 3-\mathrm{Br}, 2-\mathrm{Cl}, 3-\mathrm{Cl}, 4-\mathrm{Cl}, \\
\text { 4-F, 4-- } \mathrm{CH}_{3}, 3-\mathrm{NO}_{2}, 4-\mathrm{NO}_{2}\end{array}$ \\
\hline & $\mathrm{F}$ & 0.718 & 123.86 & -0.447 & 1.15 & 9 & $\begin{array}{c}\mathrm{H}, 3-\mathrm{Br}, 2-\mathrm{Cl}, 3-\mathrm{Cl}, 4-\mathrm{Cl} \\
\text { 4- } \mathrm{CH}_{3}, 3-\mathrm{NO}_{2}, 4-\mathrm{NO}_{2}\end{array}$ \\
\hline & $\mathrm{R}$ & 0.722 & 123.51 & -1.095 & 1.13 & 9 & $\begin{array}{l}\mathrm{H}, 3-\mathrm{Br}, 2-\mathrm{Cl}, 3-\mathrm{Cl}, 4-\mathrm{Cl}, \\
\text { 4-F, 4- } \mathrm{CH}_{3}, 3-\mathrm{NO}_{2}, 4-\mathrm{NO}_{2}\end{array}$ \\
\hline \multirow[t]{6}{*}{$\delta \mathrm{C} \beta(\mathrm{ppm})$} & $\sigma$ & 0.906 & 144.56 & 0.458 & 2.59 & 6 & $\begin{array}{c}\text { 3-Br, 2-Cl, 3-Cl, 4-Cl, } \\
\text { 4-F, 3-- } \mathrm{NO}_{2}\end{array}$ \\
\hline & $\sigma^{+}$ & 0.803 & 144.76 & -0.229 & 2.59 & 9 & $\begin{array}{c}\mathrm{H}, 3-\mathrm{Br}, 2-\mathrm{Cl}, 3-\mathrm{Cl}, 4-\mathrm{Cl}, \\
\text { 4- } \mathrm{CH}_{3}, 3-\mathrm{NO}_{2}, 4-\mathrm{NO}_{2}\end{array}$ \\
\hline & $\sigma_{\mathrm{I}}$ & 0.728 & 145.80 & -2.735 & 2.49 & 9 & $\begin{array}{c}\mathrm{H}, 3-\mathrm{Br}, 2-\mathrm{Cl}, 4-\mathrm{Cl}, 4-\mathrm{F} \\
\text { 3- } \mathrm{NO}_{2}, 4-\mathrm{NO}_{2}\end{array}$ \\
\hline & $\sigma_{\mathrm{R}}$ & 0.949 & 145.40 & 6.108 & 2.25 & 8 & $\begin{array}{c}\mathrm{H}, 3-\mathrm{Br}, 2-\mathrm{Cl}, 3-\mathrm{Cl}, 4-\mathrm{Cl}, \\
\text { 4-F, 4- } \mathrm{CH}_{3}, 3-\mathrm{NO}_{2}\end{array}$ \\
\hline & $\mathrm{F}$ & 0.839 & 145.96 & -3.046 & 2.45 & 9 & $\begin{array}{c}\mathrm{H}, 3-\mathrm{Br}, 2-\mathrm{Cl}, 3-\mathrm{Cl}, 4-\mathrm{Cl} \\
4-\mathrm{CH}_{3}, 3-\mathrm{NO}_{2}, 4-\mathrm{NO}_{2}\end{array}$ \\
\hline & $\mathrm{R}$ & 0.943 & 145.41 & 4.784 & 2.33 & 8 & $\begin{array}{c}\mathrm{H}, 3-\mathrm{Br}, 2-\mathrm{Cl}, 3-\mathrm{Cl}, 4-\mathrm{Cl} \\
\quad 4-\mathrm{F}, 4-\mathrm{CH}_{3}, 3-\mathrm{NO}_{2}\end{array}$ \\
\hline
\end{tabular}


This is due to the inductive and resonance effects of substituents incapable for predicting the reactivity on the absorption and is associated with the resonance conjugated structure as shown in Fig. 1. In single parameter correlation, the Hammett substituents constants not obeyed in the regression.

While seeking these parameters in multi-regression, with F and R Swain-Lupton's [33] constants, they gave satisfactory correlations. The multi correlation equations are given in 2 and 3.

$$
\begin{aligned}
\lambda \max (\mathrm{nm})= & 280.94( \pm 13.115)-9.738( \pm 6.290) \sigma_{\mathrm{I}}+20.359( \pm 13.619) \sigma_{\mathrm{R}} \\
& (R=0.927, \mathrm{n}=9, P>90 \%)
\end{aligned}
$$

$\lambda \max (\mathrm{nm})=286.86( \pm 11.558)-20.818( \pm 7.369) \mathrm{F}+23.726( \pm 7.587) \mathrm{R}$

$$
(R=0.947, \mathrm{n}=9, P>90 \%)
$$

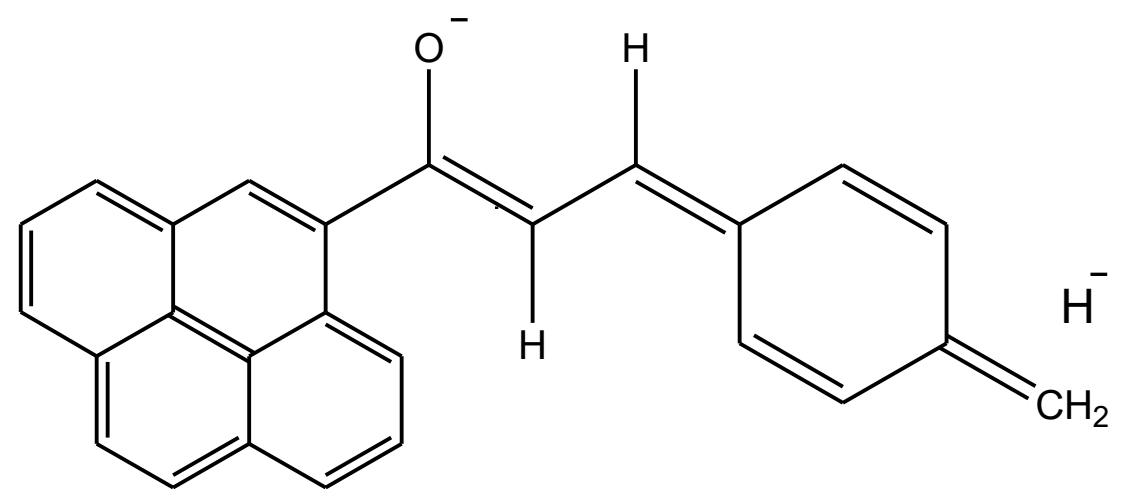

Fig. 1. The resonance - conjugated structure.

\section{2. IR spectral study}

The synthesized chalcones in the present study are shown in Scheme 1. The carbonyl stretching frequencies $\left(\mathrm{cm}^{-1}\right)$ of $s$-cis and s-trans of isomers are tabulated in in Table 2 and the corresponding conformers are shown in Fig. 2.<smiles>[X]c1ccccc1/C=C/C(=O)c1cc2cccc3ccc4cccc1c4c32</smiles>

Fig. 2. The s-cis and s-trans conformers of 1-pyrenyl chalcones.<smiles>[X]c1ccc(/C=C(\C)C(=O)c2cc3cccc4ccc5cccc2c5c43)cc1</smiles>

The stretching frequencies for carbonyl absorption are assigned based on the assignments made by Hays and Timmons [2] for s-cis and s-trans conformers at 1690 and $1670 \mathrm{~cm}^{-1}$, respectively. The assigned infrared $\mathrm{CO} s$-cis and s-trans conformers have been correlated with 
Hammett substituent constants and Swain-Lupton constants [33]. In this correlation the structure parameter Hammett equation employed is as shown in the following equation:

$$
v=\rho \sigma+v_{0}
$$

Where $v$ is the carbonyl frequencies of substituted system and $v_{0}$ is the corresponding quantity of unsubstitued system; $\sigma$ is a Hammett substituent constant, which in principle is characteristics of the substituent and $\rho$ is a reaction constant which is depend upon the nature of the reaction.

The results of statistical analysis $[1,2,6,10,19,21,23-25,28,32]$ were shown in Table 3, From table 3, The resonance component only correlated satisfactorily with $\mathrm{CO}$ s-cis conformers stretches. The remaining Hammett substituent constants and F parameters were fail in correlation. The Hammett $\sigma$ and F parameters gave satisfactory correlation with $\mathrm{CO} s$-cis conformers stretches. The remaining Hammett substituent constants and $\mathrm{R}$ parameters were fail in correlation. This is due to the reasons stated in earlier and associated with the resonance conjugative structure shown in Fig. 1.

The correlation of $\mathrm{CH}_{i p}$ modes with Hammett $\sigma_{\mathrm{R}}$ and $\mathrm{R}$ parameters gave satisfactory correlation. The remaining The remaining Hammett substituent constants and $\mathrm{F}$ parameters were fail in correlation. The Hammett $\sigma$ constants correlated satisfactorily with $\mathrm{CH}_{o p}$ modes of all chalcones. The remaining Hammett substituent constants, $\mathrm{F}$ and $\mathrm{R}$ parameters gave poor correlation. This failure in correlation is due to the incapability of polar and inductive effects of the substituents and associated with the resonance conjugative structure as shown in Fig. 1.

The Hammett $\sigma$ and $\sigma+$ constants gave satisfactory correlation with $\mathrm{CH}=\mathrm{CH}_{o p}$ modes. The inductive, resonance and field components of the substituents are failed in correlation. The Hammett $\sigma, \sigma+$ and $\sigma_{\mathrm{R}}$ constants were satisfactorily correlated with $\mathrm{C}=\mathrm{C}_{o p}$ modes. The inductive components of the substituents, $\mathrm{F}$ and $\mathrm{R}$ parameters failed in correlation. This is due to the reasons stated earlier and associated with the resonance conjugative structure as shown in Fig. 1.

Some of the single parameter correlations with Hammett substituent constants were not obeyed in the regression. While seeking these parameters in multi-regression, with $\mathrm{F}$ and $\mathrm{R}$ SwainLupton's constants [33], they gave satisfactory correlations with the infrared red group frequencies. The multi correlation equations are given in $5-16$.

$$
\begin{aligned}
& v \mathrm{CO}_{\text {s-cis }}\left(\mathrm{cm}^{-1}\right)=1649.72( \pm 17.120)+3.261( \pm 0.319) \sigma_{\mathrm{I}}+73.230( \pm 43.887) \sigma_{\mathrm{R}} \quad \ldots(5) \\
& (R=0.956, \mathrm{n}=9, P>95 \%) \\
& v \mathrm{CO}_{s-c i s}\left(\mathrm{~cm}^{-1}\right)=1650.34( \pm 17.255)+2.509( \pm 0.886) \mathrm{F}+59.230( \pm 41.184) \mathrm{R} \\
& (R=0.950, \mathrm{n}=9, P>95 \%) \\
& v \mathrm{CO}_{\text {s-trans }}\left(\mathrm{cm}^{-1}\right)=1588.82( \pm 35.872)-62.134( \pm 21.906) \sigma_{\mathrm{I}}+21.613( \pm 7.954) \sigma_{\mathrm{R}} \ldots(7) \\
& (R=0.934 \mathrm{n}=9, P>90 \%) \\
& \mathrm{vCO}_{\text {s-trans }}\left(\mathrm{cm}^{-1}\right)=1587.50( \pm 34.683)-55.091( \pm 17.120) \mathrm{F}+23.032( \pm 8.779) \mathrm{R} \\
& (R=0.933, \mathrm{n}=9, P>90 \%) \\
& v \mathrm{CH}_{i p}\left(\mathrm{~cm}^{-1}\right)=1129.09( \pm 17.324)-5.196( \pm 3.727) \sigma_{\mathrm{I}}-64.018( \pm 44.409) \sigma_{\mathrm{R}} \\
& (R=0.951, \mathrm{n}=9, P>95 \%) \\
& v \mathrm{CH}_{i p}\left(\mathrm{~cm}^{-1}\right)=1123.40( \pm 15.112)+1.049( \pm 0.553) \mathrm{F}-70.744( \pm 36.069) \mathrm{R} \\
& (R=0.963, \mathrm{n}=9, P>95 \%) \\
& { } \mathrm{CH}_{o p}\left(\mathrm{~cm}^{-1}\right)=814.03( \pm 34.300)-83.570( \pm 68.756) \sigma_{\mathrm{I}}-41.409( \pm 18.926) \sigma_{\mathrm{R}} \\
& (R=0.947, \mathrm{n}=9, P>90 \%)
\end{aligned}
$$




$$
\begin{aligned}
v \mathrm{CH}_{o p}\left(\mathrm{~cm}^{-1}\right)=806.58 & ( \pm 32.458)-78.601( \pm 65.622) \mathrm{F}-74.189( \pm 37.468) \mathrm{R} \\
(R=0.956, \mathrm{n}=9, P>95 \%) & \ldots(12) \\
v \mathrm{CH}=\mathrm{CH}_{o p}\left(\mathrm{~cm}^{-1}\right)= & 1053.56( \pm 17.991)+25.309( \pm 6.064) \sigma_{\mathrm{I}}+69.498( \pm 46.119) \sigma_{\mathrm{R}} \ldots(1) \\
(R=0.956, \mathrm{n}=9, P>95 \%) & \\
v \mathrm{CH}=\mathrm{CH}_{o p}\left(\mathrm{~cm}^{-1}\right)= & 1059.99( \pm 16.842)+16.302( \pm 4.051) \mathrm{F}+73.694( \pm 40.199) \mathrm{R} \\
(R=0.960, \mathrm{n}=9, P>95 \%) & \\
v \mathrm{C}=\mathrm{C}_{o p}\left(\mathrm{~cm}^{-1}\right)=577.29( \pm 14.497)+8.493( \pm 2.060) \sigma_{\mathrm{I}}-54.337( \pm 37.162) \sigma_{\mathrm{R}} & \ldots(15) \\
& (R=0.951, \mathrm{n}=9, P>95 \%) \\
v \mathrm{C}=\mathrm{C}_{o p}\left(\mathrm{~cm}^{-1}\right)=574.75( \pm 13.424)+11.380( \pm 7.144) \mathrm{F}-50.538( \pm 32.041) \mathrm{R} & \ldots(16) \\
& (R=0.957, \mathrm{n}=9, P>95 \%)
\end{aligned}
$$

\section{3. NMR Spectral study}

\section{3. $1 .{ }^{1} \mathrm{H}$ NMR spectra}

From the ${ }^{1} \mathrm{H}$ NMR spectra of chalcones the chemical shifts ( $\delta$, ppm ) $\mathrm{H} \alpha$ and $\mathrm{H} \beta$ are assigned and tabulated in Table 2 . These chemical shifts were correlated with Hammett substituent constants, $\mathrm{F}$ and $\mathrm{R}$ parameters. The statistical analysis $[1,2,6,10,19,21,23-25,28,32]$ of these chemical shifts is presented in Table 3. From Table 3, the H $\alpha$ chemical shifts $(\delta, \mathrm{ppm})$ correlated with Hammett $\sigma, \sigma^{+}, \sigma_{R}$ constants and $\mathrm{R}$ parameters. The inductive and Field effects of the substituents were fail in correlations. Hammett $\sigma$ and $\sigma^{+}$constants gave satisfactory correlation with $\mathrm{H} \alpha$ chemical shifts $(\delta, \mathrm{ppm})$. The inductive, field and resonance components of the substituents fail in correlation. The failure in correlation for both chemical shifts are the reasons stated earlier and associated with the resonance - conjugated structure shown in Fig. 1.

Some of the single parameter correlations with Hammett substituent constants were not obeyed in the regression. While seeking these parameters in multi-regression, with $\mathrm{F}$ and $\mathrm{R}$ SwainLupton's [33] constants, they gave satisfactory correlations with the chemical shifts of ( $\delta, \mathrm{ppm}) \mathrm{H} \alpha$ and $\mathrm{H} \beta$. The multi correlation equations are given in 17-20.

$$
\begin{aligned}
\delta \mathrm{H}_{\alpha}(\mathrm{ppm})= & 7.530( \pm 0.221)+0.069( \pm 0.044) \sigma_{\mathrm{I}}+0.826( \pm 0.566) \sigma_{\mathrm{R}} \\
& (R=0.951, \mathrm{n}=9, P>95 \%) \\
\delta \mathrm{H}_{\alpha}(\mathrm{ppm})= & 7.562( \pm 0.218)+0.006( \pm 0.004) \mathrm{F}+0.693( \pm 0.521) \mathrm{R} \\
& (R=0.948, \mathrm{n}=9, P>90 \%) \\
\delta \mathrm{H}_{\beta}(\mathrm{ppm})= & 7.950( \pm 0.200)+0.382( \pm 0.040) \sigma_{\mathrm{I}}+0.575( \pm 0.051) \sigma_{\mathrm{R}} \\
& (R=0.952, \mathrm{n}=9, P>95 \%) \\
\delta \mathrm{H}_{\beta}(\mathrm{ppm})= & 8.019( \pm 0.195)+0.271( \pm 0.039) \mathrm{F}+0.624( \pm 0.467) \mathrm{R} \\
& (R=0.950, \mathrm{n}=9, P>95 \%)
\end{aligned}
$$

\section{3. $1 .{ }^{13} \mathrm{C}$ NMR spectra}

The carbonyl carbon, $\mathrm{C} \alpha$ and $\mathrm{C} \beta$ chemical shifts ( $\delta$, ppm)of 1-pyrenyl chalcones were assigned and tabulated in Table 2. These chemical shifts are correlated $[1,2,6,10,19,21,23-25$, 28,32] with Hammett substituent constants, $\mathrm{F}$ and $\mathrm{R}$ parameters. The results of statistical analysis are shown in Table 3. From Table 3, the Hammett $\sigma, \sigma^{+}, \sigma_{\mathrm{I}}$ constants and F parameters were gave satisfactory correlations with carbonyl carbon chemical shifts of chalcones. The Hammett $\sigma_{\mathrm{I}}$ constants correlated satisfactorily with $\mathrm{C} \alpha$ chemical shifts. The $\mathrm{C} \beta$ chemical shifts $(\delta, \mathrm{ppm})$ were satisfactorily correlated with the Hammett $\sigma, \sigma_{\mathrm{R}}$ constants and R parameters. The remaining 
Hammett substituent constants and $\mathrm{F}$ or $\mathrm{R}$ parameters were fail in correlations. This is due to the reasons stated in earlier and associated with the resonance conjugative structure shown in Fg. 1.

The multi regression analysis of carbonyl carbon, $C \alpha$ and $C \beta$ chemical shifts $(\delta$, ppm) of 1pyrenyl chalcones were satisfactorily correlated with $\sigma_{I}, \sigma_{R}$ and Swain-Luptons'[33] $F$ and $R$ parameters. The multi-regression equation are given in 21-26.

$$
\begin{aligned}
& \delta \mathrm{CO}(\mathrm{ppm})= 197.17( \pm 1.355)-3.533( \pm 2.716) \sigma_{\mathrm{I}}+7.671( \pm 3.474) \sigma_{\mathrm{R}} \\
&(R=0.971, \mathrm{n}=9, P>95 \%) \\
& \delta \mathrm{CO}(\mathrm{ppm})= 197.77( \pm 1.009)-4.645( \pm 2.040) \mathrm{F}+6.606( \pm 2.408) \mathrm{R} \\
&(R=0.984, \mathrm{n}=9, P>95 \%) \\
& \delta \mathrm{C}_{\alpha}(\mathrm{ppm})=123.86( \pm 0.823)-0.875( \pm 0.650) \sigma_{\mathrm{I}}-1.458( \pm 0.110) \sigma_{\mathrm{R}} \\
&(R=0.934, \mathrm{n}=9, P>90 \%) \\
& \delta \mathrm{C}_{\alpha}(\mathrm{ppm})=123.74( \pm 0.815)-0.595( \pm 0.064) \mathrm{F}-1.197( \pm 0.945) \mathrm{R} \\
&(R=0.926, \mathrm{n}=9, P>90 \%) \\
& \delta \mathrm{C}_{\beta}(\mathrm{ppm})=146.60( \pm 1.588)-2.900( \pm 1.184) \sigma_{\mathrm{I}}+6.233( \pm 4.072) \sigma_{\mathrm{R}}(R=0.958, \mathrm{n}=9, P>95 \%) \\
& \delta \mathrm{C}_{\beta}(\mathrm{ppm})=146.39( \pm 1.619)-2.511( \pm 0.273) \mathrm{F}+4.355( \pm 3.864) \mathrm{R} \\
&(R=0.951, \mathrm{n}=9, P>95 \%)
\end{aligned}
$$

\section{4. Antimicrobial activities}

Chalcones possess various biological activities such as antibacterial [26,34], antifungal [26], antioxidant [8], antiviral [9], antimalarial [10], antiplasmodial [28], antituberclosis [12], antiproliferative [13], antileshmanial [14], anti-inflammatory [15], antianalgesic and sedative [16], insect antifeedants [13,22]. Halogenated chalcones possess insect antifeedant activities [13,22,42]. The presence of a reactive $\alpha, \beta$-unsaturated keto function in chalcones was found to be responsible for their antimicrobial activity. At present, scientists have paid more interest to correlate the group frequencies of spectral data with Hammett substituent constants to explain the substituent effects of organic compounds.

\section{4. 1. Antibacterial sensitivity assay}

Antibacterial sensitivity assay was performed using Kirby-Bauer [30] disc diffusion technique. In each Petri plate about $0.5 \mathrm{~mL}$ of the test bacterial sample was spread uniformly over the solidified Mueller Hinton agar using sterile glass spreader.

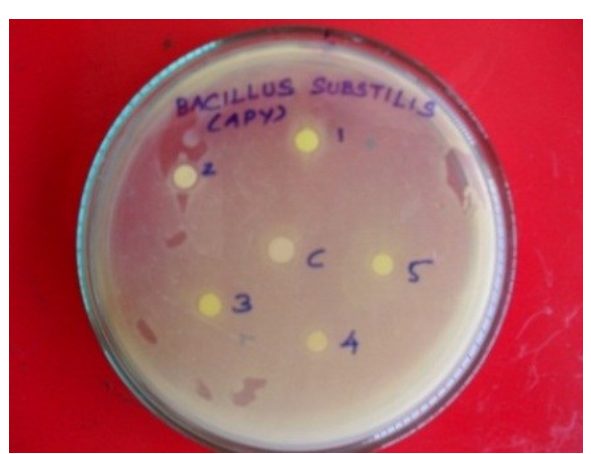

Plate-1

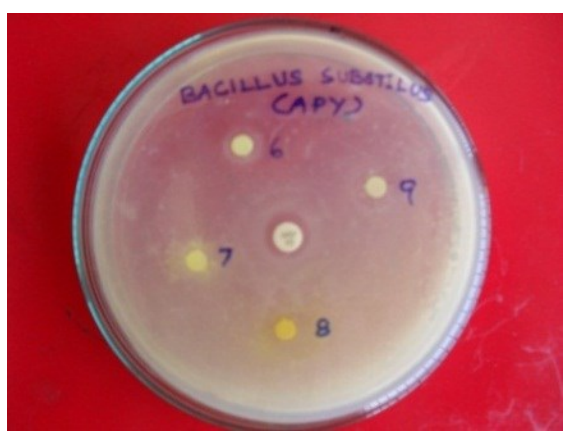

Plate-2 


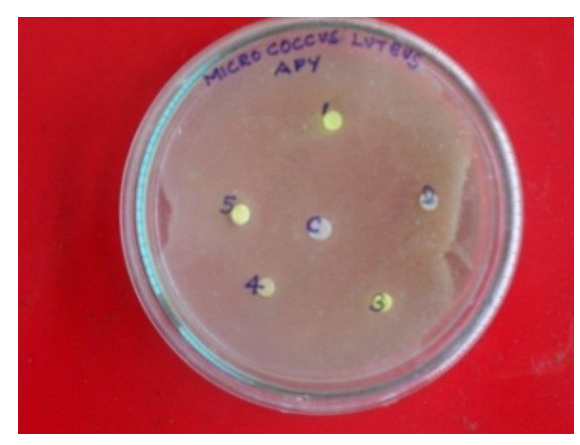

Plate-3

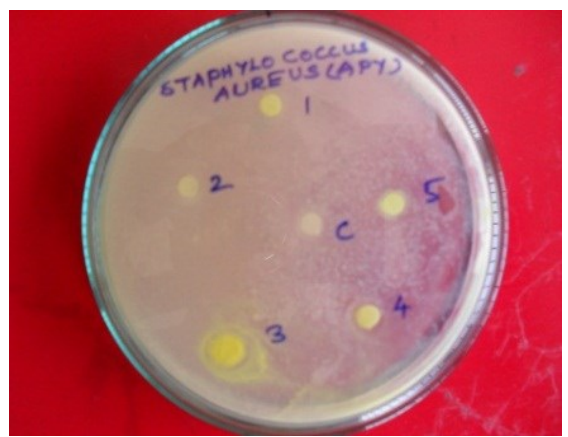

Plate-5

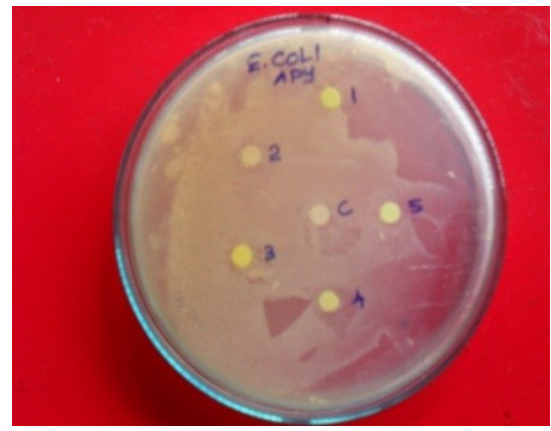

Plate-7

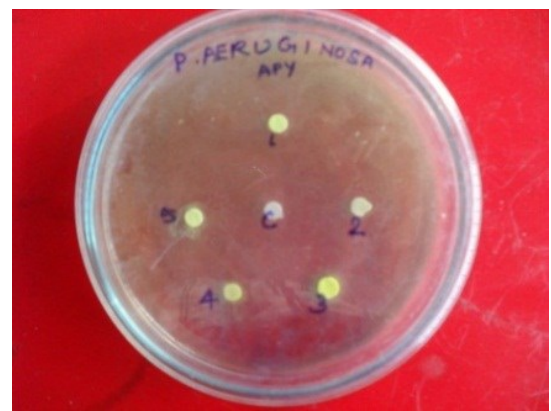

Plate-9

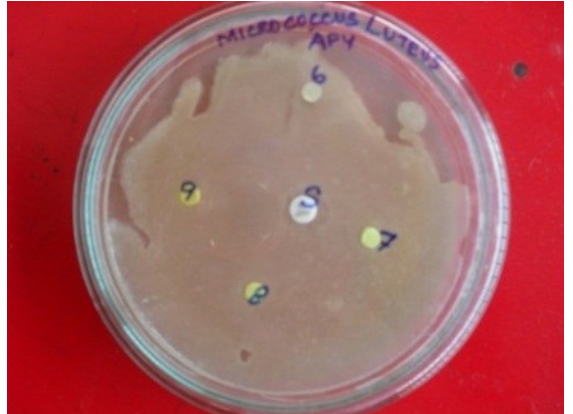

Plate-4

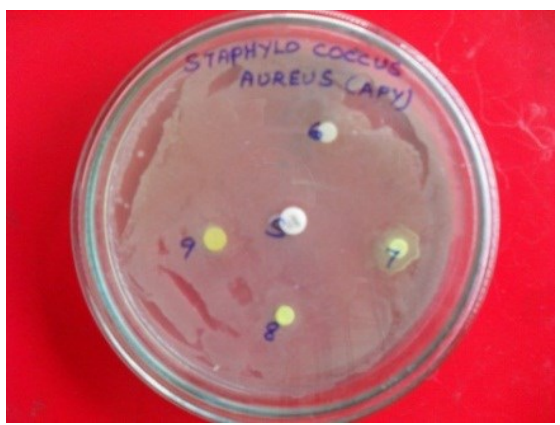

Plate-6

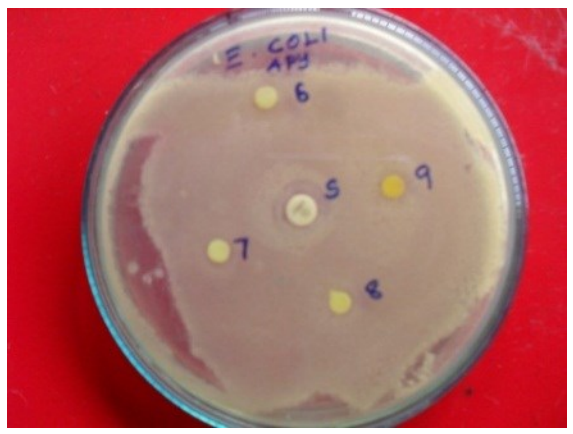

Plate-8

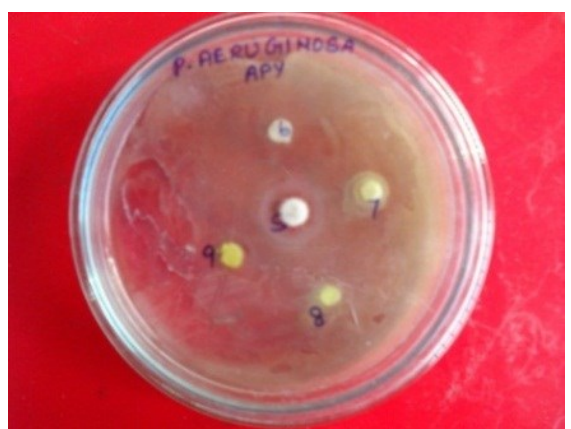

Plate-10

Fig. 3. Antibacterial activities of substituted styryl 1-pyrenyl ketones.

Then the discs with $5 \mathrm{~mm}$ diameter made up of Whatmann No.1 filter paper, impregnated with the solution of the compound were placed on the medium using sterile foreceps. The plates were incubated for 24 hours at $37^{\circ} \mathrm{C}$ by keeping the plates upside down to prevent the collection of water droplets over the medium. After 24 hours, the plates were visually examined and the diameter values of the zone of inhibition were measured. Triplicate results were recorded by repeating the same procedure. The antibacterial effect of the styryl 1-pyrenyl ketones is shown in Fig. 3 for Plates 
(1)-(10). Analysis of the zone of inhibition as given Table-4 and the Clustered column Chart Fig 4, reveals that all the substituents except $3-\mathrm{Br}$ have shown moderate antibacterial activity against all the bacterial species, under investigation. The substituent 3- $\mathrm{NO}_{2}$ has shown more antibacterial activity than standard (Ampicillin) against B. subtilis species. The substituent 2-Cl has shown more antibacterial activity than standard (Ampicillin) against $S$. aureus species. The substituent $4-\mathrm{Cl}$ has shown equal antibacterial activity with standard (Ampicillin) against $M$. luteus species. The parent compound $(\mathrm{H})$ has shown equal activities against all bacteria except $P$. aeruginosa. The compound with 2-Cl, 3-Cl and 4-Cl substituents has shown equal activities against $B$. subtilis bacterial species. The compound with $3-\mathrm{NO}_{2}$ and $4-\mathrm{NO}_{2}$ substituents has shown equal activities against $M$. luteus and S. aureus bacterial species.

\section{4. 2. Antifungal sensitivity assay}

Antifungal sensitivity assay was performed using Kirby-Bauer [30] disc diffusion technique. PDA medium was prepared and sterilized as above. It was poured (ear bearing heating condition) in the Petri-plate which was already filled with $1 \mathrm{ml}$ of the fungal species.

The plate was rotated clockwise and counter clock-wise for uniform spreading of the species. The discs were impregnated with the test solution.

Table 4. Antibacterial activity of substituted styryl 1-pyrenyl ketones

\begin{tabular}{|c|c|c|c|c|c|c|}
\hline \multirow{2}{*}{ Entry } & \multirow{2}{*}{ Substt. } & \multicolumn{5}{|c|}{ Zone of Inhibition (mm) } \\
\cline { 3 - 7 } & & \multicolumn{2}{|c|}{ Gram positive Bacteria } & \multicolumn{2}{c|}{ Gram negative Bacteria } \\
\cline { 3 - 7 } & & B. subtilis & M. luteus & S. aureus & E. coli & P. aeruginosa \\
\hline 1 & $\mathrm{H}$ & 7 & 7 & 7 & 7 & 6 \\
\hline 2 & $3-\mathrm{Br}$ & 6 & 7 & 6 & - & 7 \\
\hline 3 & $2-\mathrm{Cl}$ & 6 & 6 & 9 & 6 & 7 \\
\hline 4 & $3-\mathrm{Cl}$ & 6 & 7 & 6 & 7 & 6 \\
\hline 5 & $4-\mathrm{Cl}$ & 6 & 9 & 6 & 6 & 7 \\
\hline 6 & $4-\mathrm{F}$ & 7 & 6 & 7 & 6 & 6 \\
\hline 7 & $4-\mathrm{CH}_{3}$ & 7 & 7 & 8 & 6 & 8 \\
\hline 8 & $3-\mathrm{NO}_{2}$ & 9 & 6 & 6 & 7 & 6 \\
\hline 9 & $4-\mathrm{NO}_{2}$ & 6 & 6 & 6 & 8 & 7 \\
\hline Standard & Ampicillin & 8 & 9 & 8 & 9 & 10 \\
\hline Control & $\mathrm{DMSO}^{*}$ & - & - & - & - & - \\
\hline
\end{tabular}




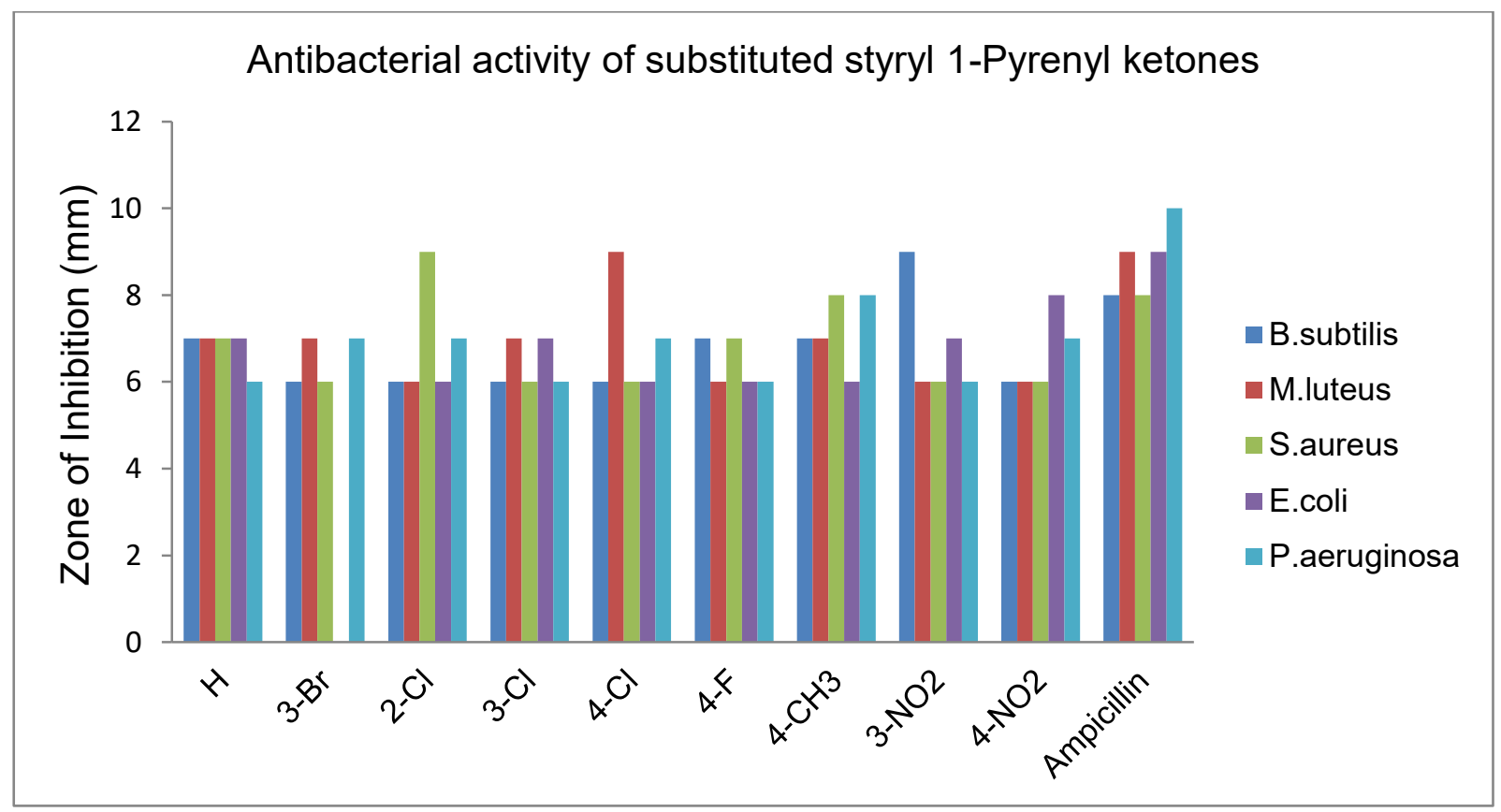

Fig. 4. Antibacterial activity of substituted styryl 1-pyrenyl ketone.

The test solution was prepared by dissolving $15 \mathrm{mg}$ of the Chalcone in $1 \mathrm{~mL}$ of DMSO solvent. The medium was allowed to solidify and kept for 24 hours. Then the plates were visually examined and the diameter values of zone of inhibition were measured. Triplicate results were recorded by repeating the same procedure. The antifungal effect of the styryl 1-pyrene ketones is shown in Fig. 5 for Plates (1-6). Analysis of the zone of inhibition as given Table 5 and the Clustered column Chart Fig 6, reveals that all the parent compound, 3-Cl and 3- $\mathrm{NO}_{2}$ substituents have shown moderate antifungal activities against all the fungal species namely $A$. niger, $P$. scup and $T$. viride, under investigation. The 2- $\mathrm{Cl}$ and $4-\mathrm{CH}_{3}$ substituents of this have shown inactive for the above three fungal species. The parent compound $(\mathrm{H})$ and $3-\mathrm{Cl}$ substituted compound has shown equal activity against all fungal species namely A. niger, P. scup and T. viride.

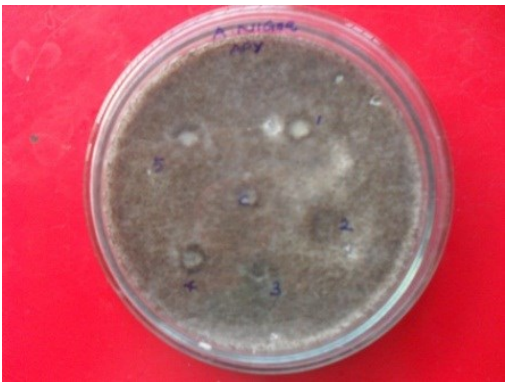

Plate-1

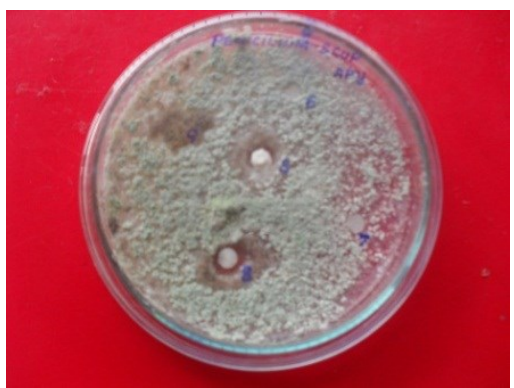

Plate-4

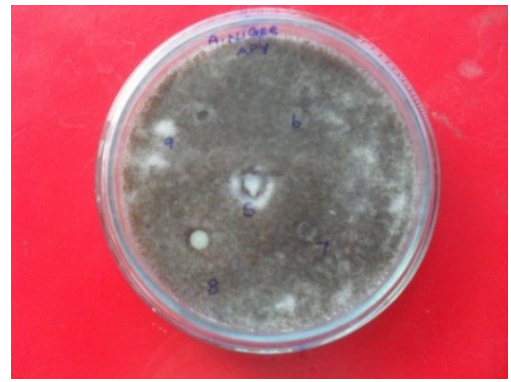

Plate-2

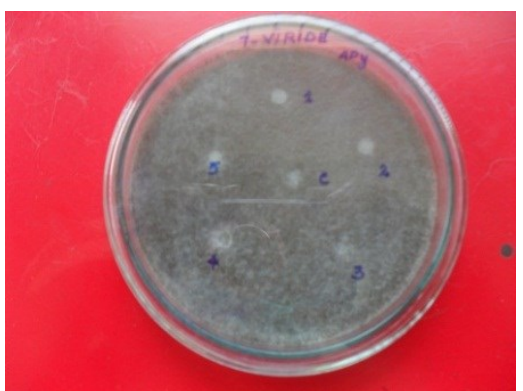

Plate-5

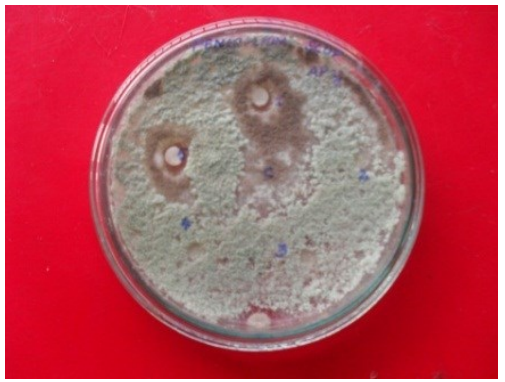

Plate-3

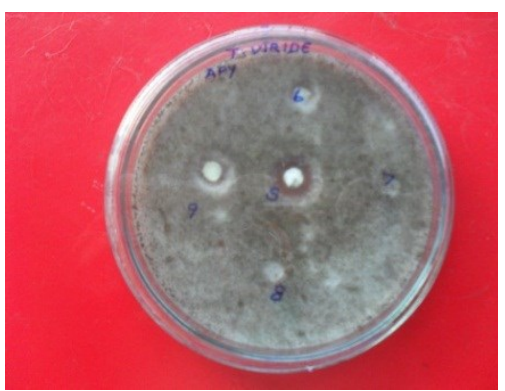

Plate-6

Fig. 5. Antifungal activities substituted styryl 1-pyrenyl ketones. 
Table 5. Antifungal activity of substituted styryl 1-pyrenyl ketones

\begin{tabular}{|c|c|c|c|c|}
\hline \multirow{2}{*}{ Entry } & \multirow{2}{*}{ Substt. } & \multicolumn{3}{|c|}{ Zone of Inhibition (mm) } \\
\cline { 3 - 5 } & & $\boldsymbol{A}$. niger & $\boldsymbol{P}$. $\boldsymbol{s p p}$ & $\boldsymbol{T}$. viride \\
\hline 1 & $\mathrm{H}$ & 6 & 6 & 6 \\
\hline 2 & $3-\mathrm{Br}$ & - & - & 6 \\
\hline 3 & $2-\mathrm{Cl}$ & - & - & - \\
\hline 4 & $3-\mathrm{Cl}$ & 6 & 6 & 6 \\
\hline 5 & $4-\mathrm{Cl}$ & 6 & 7 & - \\
\hline 6 & $4-\mathrm{F}$ & - & 6 & 6 \\
\hline 7 & $4-\mathrm{CH}_{3}$ & - & - & - \\
\hline 8 & $3-\mathrm{NO}_{2}$ & 6 & 9 & 6 \\
\hline 9 & $4-\mathrm{NO}_{2}$ & - & - & 7 \\
\hline Standard & Miconazole & 10 & 10 & 10 \\
\hline control & DMSO & - & - & - \\
\hline
\end{tabular}

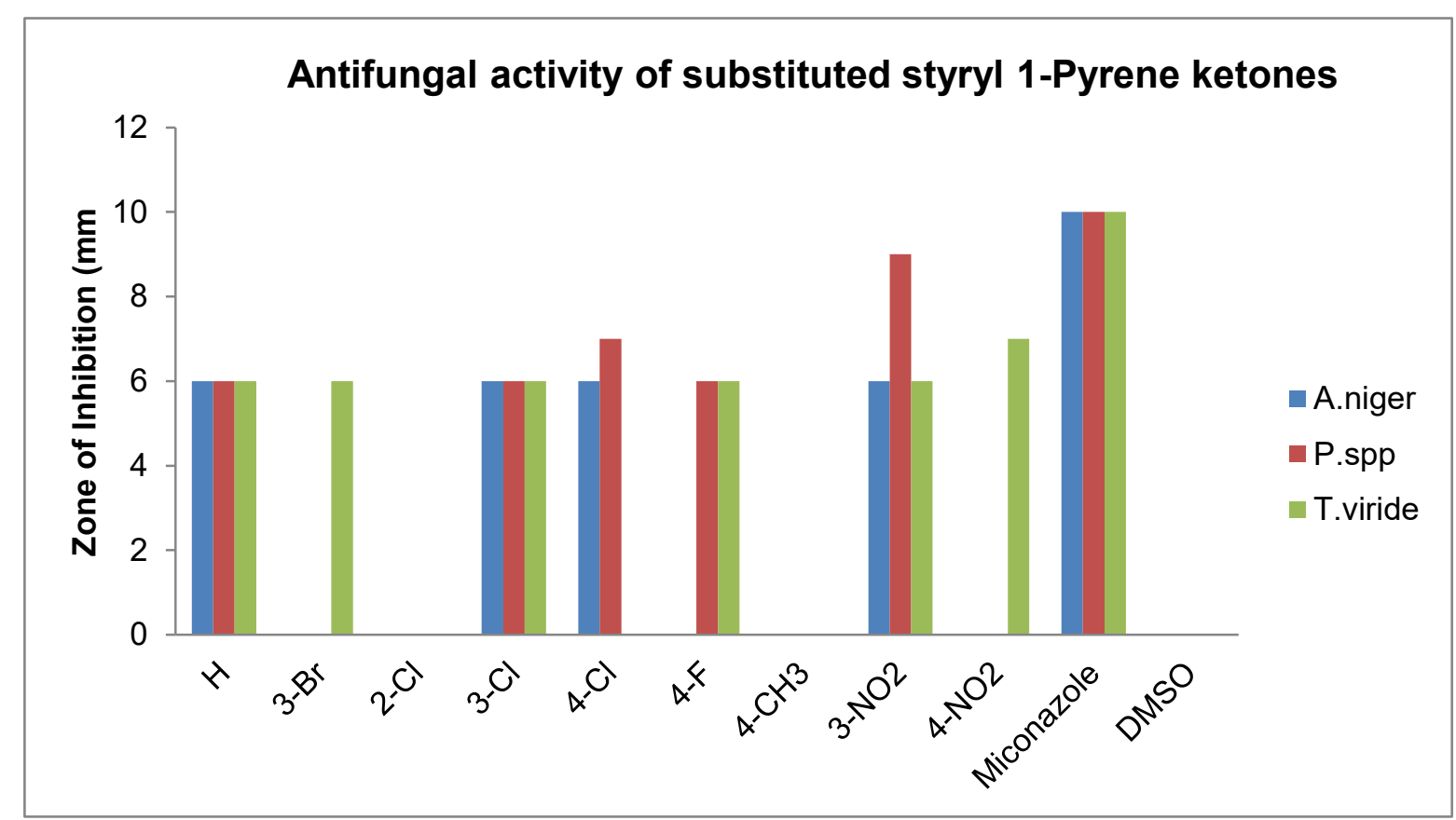

Fig. 6. Antifungal activity of substituted styryl 1-pyrene ketones-clustered column chart.

\section{CONCLUSIONS}

A series of 1-pyrenyl chalcones have been synthesized by Crossed-Aldol condensation. The spectral data of these chalcones have been correlated with Hammett sigma constants, F and R parameters using single and multi-linear regression analysis. From the results of statistical analysis, the effects of substituents on the spectral group frequencies have been discussed. The antimicrobial activities of these chalcones have been studied 


\section{Acknowledgement}

The authors thank DST NMR facility, Department of Chemistry, Annamalai University, Annamalainagar-608 002, for recording NMR spectra of all compounds.

\section{REFERENCES}

[1] Thirunarayanan G., Vanangamudi G., Subramanian M., Organic Chem: An Indian J. 9(1), (2013) 1-16.

[2] Janaki P., Sekar K. G., Thirunarayanan G., J. Saudi Chem. Soc., 2013, Accepted and in press. DOI: org/10.1016/j.jscs.2012.11.013.

[3] Holla B. S., Akberali P. M., Shivananda M. K., IL Farmaco 55 (2000) 256-261.

[4] Venkat Reddy G., Maitraie D., Narsaiah B., Rambabu Y., Shanthan Rao P., Synth. Commun 31(18), (2004) 2882-2884.

[5] Orsini F., Sello G., Fumagalli T., Synlett. 11 (2006) 1717-1718.

[6] Vanangamudi G., Subramanian S., Jayanthi P., Arulkumaran R., Kamalakkannan D., Thirunarayanan G., Arab. J. Chem., 2010, DOI: 10.1016/j.arabjc.2010.07.019.

[7] Tran T. D., Park H., Ecker G. F., Thai K. M., 12 ${ }^{\text {th }}$ International Electronic Conference on Synthetic Organic Chemistry (ECSOC-12), 1-30 November 2008; http://www.usc.es/congresos/ecsoc/12/ECSOC12.htm \& http://www.mdpi.org/ecsoc-12 No. C0011, pp 1-7.

[8] Mohan S. B., Behera T. P., Ravi Kumar B. V. V., Int. J. ChemTech. Res. 2(3), (2010) 1634-1637.

[9] Thirunarayanan G., Vanangamudi G., Arkivoc. 12 (2006) 58-64.

[10] Thirunarayanan G., Ananthakrishna Nadar P., J. Indian Chem. Soc. 83(11), (2006) 11071112.

[11] Ballini R., Bosica G., Maggi R., Ricciutelli M., Righi P., Sartori G., Sartorio R., Green Chem. 3 (2001) 178-180 .

[12] Solhy A., Tahir R., Sebti S., Skouta R., Bousmina M., Zahouily M., Larzek M., Appl. Catal. A: General. 374 (2010) 189-193

[13] Thirunarayanan G., J. Indian Chem. Soc. 84 (2008) 447-451.

[14] Basaif S. A., Sobahi T. R., Khalil A. K., Hassan M. A., Bulletin of the Korean Chem. Soc. 26(11) (2005) 1677-1681.

[15] Xu Q., Yang Z., Yin D., Zhang F., Catal. Commun. 9(1) (2008) 1579-1582.

[16] Kumar P., Kumar S., Husain K., Kumar A., Bioorg. Med. Chem. 18(14) (2010) 49654974.

[17] Zhang Z., Dong Y. W., Wang G. W., Chem. Lett. 32(10), (2003) 966-967.

[18] Thirunarayanan G., IUP. J. Chem. 3(4) (2010) 35-54. 
[19] Thirunarayanan G., Thirumurthy K., Vanangamudi G., Subramanian M., Arulkumaran R., Kamalakkannan D., Sundararajan R., Sakthinathan S. P., Vijayakumar S., Ranganathan K., Suresh R., Elixir Org. Chem. 45 (2012) 7898-7905.

[20] Thirunarayanan G., Mayavel P., Thirumurthy K., Spectrochimica Acta. 91A (2012) 18-22.

[21] Sundararajan R., Arulkumaran R., Vijayakumar S., Kamalakkannan D., Suresh R., Ranganathan K., Sakthinathan S. P., Vanangamudi G., Thirumurthy K., Mayavel P., Thirunarayanan G., Int. J. Pharm. Chem. Sci. 1(4) (2012) 1657-1677.

[22] Thirunarayanan G., Surya S., Srinivasan S., Vanangamudi G., Sathiyendiran V., Spectrochim. Acta. 75A (2010) 152-156.

[23] Janaki P., Sekar K. G., Thirunarayanan G., Org. Chem: An Indian J. 9(2) (2013) 68-80.

[24] Arulkumaran R., Vijayakumar S., Sundararajan R., Sakthinathan S. P., Kamalakkannan D., Suresh R., Ranganathan K., Vanangamudi G., Thirunarayanan G., Int. Lett. Chem. Phys. Astro. 4 (2012) 17-38.

[25] Kamalakkannan D., Vanangamudi G., Arulkumaran R., Thirumurthy K., Mayavel P., Thirunarayanan G., Elixir Org. Chem. 46 (2012) 8157-8166.

[26] Sivakumar M., Phrabu Sreeneivasan S., Kumar V., Doble M., Bioorg. Med. Chem. Lett. 17(10) (2007) 3169-3172.

[27] Liu X., Go M. L., Bioorg. Med. Chem. 14(1) (2006) 153-163.

[28] Arulkumaran R., Sundararajan R., Vanangamudi G., Subramanian M., Ravi K., Sathiyendidran V., Srinivasan S., Thirunarayanan G., IUP J. Chem. 3(1) (2010) 82-98.

[29] Deng J., Sanchez T., Lalith Q. A. M., Bioorg, Med. Chem. 15(14) (2007) 4985-5002.

[30] Bauer A. W., Kirby W. M. M., Sherris J. C., Truck M., Am. J. Clin. Pathol. 45 (1966) 493-496.

[31] Thirunarayanan G., "Fly-ash: $\mathrm{H}_{2} \mathrm{O}$ Assisted Aldol Condensation: Effect of Solvents on the Synthesis of Some Aryl (E) 2-propen-1-ones" Accepted article in Annales, UMCS Chem. J., 2013.

[32] Ranganathan K., Suresh R., Kamalakkannan D., Arulkumaran R., Sundararajan R., Sakthinathan S. P., Vijayakumar S., Vanangamudi G., Thirumurthy K., Mayavel P., Thirunarayanan G., Int. Lett. Chem. Phys. Astro. 4 (2012) 66-75.

[33] Swain C. G., Lupton Jr. E. C., J. Am. Chem. Soc. 90(16) (1968) 4328-4337.

[34] Deiva C. M., Pappano N. B., Debattisata N. B., Rev. Microbiol. 29(4) (1998) 307-310.

[35] Lahtchev K. L., Batovska D. I., St Parushev P., Ubiyvock V. M., Sibirny A. A., Eur. J. Med. Chem. 43(1) (2008) 2220-2228.

[36] Weber M. W, Hunsaker L. A., Abcouwer S. F., Decker L. M., Vander D. L. Jagat, Bioorg. Med. Chem. 13 (2005) 3811-3820.

[37] Dominguez J. N., Leon C., Rodrigues J., IL Farmaco. 60(4) (2005) 307-311.

[38] Lin Y. M., Zhon Y., Flavin M. T., Zhon L. M., Ne W., Chen F. C., Bioorg. Med. Chem. 10 (8) (2002) 2795-2802.

[39] Liu X., Go M. L., Bioorg. Med. Chem. 14 (2006) 153-163. 
[40] Delmulle L., Bellahcene A., Dhooge W., Comhaire F., Roelens F., Huvaere K., Heyerick A., Castronovo V., Keukeleire D. D., Phytomed. 13 (2006) 732-734.

[41] Nielsen S. F., Chen M., Theander T. G., Kharazmi A., Christensen S. B., Bioorg. Med. Chem. Lett. 5 (1995) 449-452.

[42] Thirunarayanan G., J. Saudi Chem. Soc., 2011. DOI:10.1016/j.jscs.2011.12.003. 